\title{
CONVERGENCE OF NUMERICAL METHODS FOR STOCHASTIC DIFFERENTIAL EQUATIONS IN MATHEMATICAL FINANCE
}

\author{
PETER KLOEDEN AND ANDREAS NEUENKIRCH
}

\begin{abstract}
Many stochastic differential equations that occur in financial modelling do not satisfy the standard assumptions made in convergence proofs of numerical schemes that are given in textbooks, i.e., their coefficients and the corresponding derivatives appearing in the proofs are not uniformly bounded and hence, in particular, not globally Lipschitz. Specific examples are the Heston and Cox-Ingersoll-Ross models with square root coefficients and the Ait-Sahalia model with rational coefficient functions. Simple examples show that, for example, the Euler-Maruyama scheme may not converge either in the strong or weak sense when the standard assumptions do not hold. Nevertheless, new convergence results have been obtained recently for many such models in financial mathematics. These are reviewed here. Although weak convergence is of traditional importance in financial mathematics with its emphasis on expectations of functionals of the solutions, strong convergence plays a crucial role in Multi Level Monte Carlo methods, so it and also pathwise convergence will be considered along with methods which preserve the positivity of the solutions.
\end{abstract}

\section{INTRODUCTION}

Consider the Itô stochastic differential equation $(\mathrm{SDE})$ in $\mathbb{R}^{d}$

$$
d X_{t}=a\left(X_{t}\right) d t+\sum_{j=1}^{m} b_{j}\left(X_{t}\right) d W_{t}^{(j)}, \quad t \in[0, T], \quad X_{0}=x_{0} \in \mathbb{R}^{d}
$$

with drift and diffusion coefficients $a, b_{j}: \mathbb{R}^{d} \rightarrow \mathbb{R}^{d}$ for $j=1, \ldots, m$. Here $W_{t}=$ $\left(W_{t}^{(1)}, \ldots, W_{t}^{(m)}\right), t \geq 0$, is an $m$-dimensional Brownian motion on a probability space $(\Omega, \mathcal{F}, \mathbf{P})$ and superscripts in brackets label components of vectors. Throughout this article it will always be assumed that equation (1) has a unique strong solution.

Explicit solutions of such equations are rarely known, thus one has to rely on numerical methods to simulate their sample paths $X_{t}(\omega)$ or to estimate functionals $\mathbf{E} \Phi(X)$ for some $\Phi: C\left([0, T] ; \mathbb{R}^{d}\right) \rightarrow \mathbb{R}$. Typically, such a numerical method relies on a discretization

$$
0 \leq t_{1} \leq t_{2} \leq \ldots \leq t_{n}=T
$$

and a global approximation on $[0, T]$ is obtained by interpolation.

In the case of the classical weak approximation the error of an approximation $\bar{X}$ to $X$ is measured by the quantity

$$
\left|\mathbf{E} \phi\left(X_{T}\right)-\mathbf{E} \phi\left(\bar{X}_{T}\right)\right|
$$

for smooth functions $\phi: \mathbb{R}^{d} \rightarrow \mathbb{R}$. The test functions $\phi$ are a particular case of the general (path-dependent) functionals $\Phi$. In the strong approximation problem the $p$-th

Date: May 1, 2012. 
mean of the difference between $X$ and $\bar{X}$ is analyzed, i.e.

$$
\left(\mathbf{E} \sup _{k=0, \ldots, n}\left|X_{t_{k}}-\bar{X}_{t_{k}}\right|^{p}\right)^{1 / p}
$$

for the maximal error in the discretization points or

$$
\left(\mathbf{E} \sup _{t \in[0, T]}\left|X_{t}-\bar{X}_{t}\right|^{p}\right)^{1 / p}
$$

for the global error, where $p \geq 1$ and $|\cdot|$ denotes the Euclidean norm. Here the meansquare error, i.e. $p=2$, is usually studied. The recent development of the Multi-level Monte Carlo method for SDEs [19, 20] has revealed that strong error bounds are crucial for the efficient computation of functionals $\mathbf{E} \Phi(X)$.

While the strong error measures the error of the approximate sample paths $\bar{X}$ on average, the pathwise error is the random quantity

$$
\sup _{k=0, \ldots, n}\left|X_{t_{k}}(\omega)-\bar{X}_{t_{k}}(\omega)\right|, \quad \omega \in \Omega
$$

and

$$
\sup _{t \in[0, T]}\left|X_{t}(\omega)-\bar{X}_{t}(\omega)\right|, \quad \omega \in \Omega
$$

respectively. Here the error is analyzed for a fixed $\omega \in \Omega$ without averaging. This quantity thus gives the error of the actually calculated approximation $\bar{X}_{t_{k}}(\omega), k=$ $0, \ldots, n$, respectively $\bar{X}(\omega)$.

The traditional weak and strong convergence analysis for numerical methods for stochastic differential equations (SDEs) relies on the global Lipschitz assumption, i.e. the SDE coefficients satisfy

$$
|a(x)-a(y)|+\sum_{j=1}^{m}\left|b_{j}(x)-b_{j}(y)\right| \leq L \cdot|x-y|, \quad x, y \in \mathbb{R}^{d}
$$

for some $L>0$. However, in many SDEs used for modelling in mathematical finance this assumption is violated, so the standard results (see [39, 41]) do not apply.

The Constant Elasticity of Variance Model for asset prices [12], which was introduced by Cox in 1975, is given by the SDE

$$
d S_{t}=\mu S_{t} d t+\sigma S_{t}^{\gamma} d W_{t}, \quad S_{0}=s_{0}>0
$$

where $\mu \in \mathbb{R}, \sigma>0$ and $\gamma \in(0,1]$ and $W_{t}, t \geq 0$, is a one-dimensional Brownian motion. For $\gamma=1$ this is the standard Black-Scholes model (i.e. a geometric Brownian motion), while for $\gamma \in(0,1)$ the diffusion coefficient of this SDE is clearly not globally Lipschitz continuous. This SDE has a unique strong solution if and only if $\gamma \in[1 / 2,1]$ and takes values in $[0, \infty)$.

The Ait-Sahalia model and its generalization [1, 45], which are stochastic interest rate models, follow the dynamics

$$
d X_{t}=\left(\alpha_{-1} X_{t}^{-1}-\alpha_{0}+\alpha_{1} X_{t}-\alpha_{2} X_{t}^{r}\right) d t+\sigma X_{t}^{\rho} d W_{t}, \quad X_{0}=x_{0}>0
$$

where $\alpha_{i}, \sigma, r, \rho>0, i=-1, \ldots, 2$. Under certain conditions on the parameters (see [45]), this SDE has a unique strong solution with values in $(0, \infty)$. Note that here the 
diffusion coefficient grows superlinearly for large values of $x$ while the drift coefficient has a singularity at $x=0$.

The Heston model [26], which is an asset price model with stochastic volatility, is another example for an SDE with non-Lipschitz coefficients. This SDE takes non-negative values only and contains square root coefficients:

$$
\begin{array}{rlrl}
d S_{t} & =\mu S_{t} d t+\sqrt{V_{t}} S_{t}\left(\sqrt{1-\rho^{2}} d W_{t}^{(1)}+\rho d W_{t}^{(2)}\right), & & S_{0}=s_{0}>0 \\
d V_{t} & =\kappa\left(\lambda-V_{t}\right) d t+\theta \sqrt{V_{t}} d W_{t}^{(2)}, & V_{0}=v_{0}>0 .
\end{array}
$$

The parameters satisfy $\mu \in \mathbb{R}, \kappa, \lambda, \theta>0$ and $\rho \in(-1,1)$. The second component of this SDE is the Cox-Ingersoll-Ross process, which is also used as a short rate model [13].

Finally, the use of the inverse of the CIR process as volatility process leads to the so-called 3/2-model

$$
\begin{array}{ll}
d S_{t}=\mu S_{t} d t+\sqrt{V_{t}} S_{t}\left(\sqrt{1-\rho^{2}} d W_{t}^{(1)}+\rho d W_{t}^{(2)}\right), & S_{0}=s_{0}>0 \\
d V_{t}=c_{1} V_{t}\left(c_{2}-V_{t}\right) d t+c_{3} V_{t}^{3 / 2} d W_{t}^{(2)}, & V_{0}=v_{0}>0
\end{array}
$$

where $c_{1}, c_{2}, c_{3}>0$, see e.g. [27].

Motivated by these and other examples, the investigation of numerical methods for SDEs with non-Lipschitz coefficients has been an active field of research in recent years. This article, provides an overview of the new developments using the above equations as illustrative examples and discussing, in particular, Euler-type schemes. For some of the above equations exact simulation methods exist, see e.g. [11, 22 and also [9] for a class of one-dimensional equations, which are superior for the simulation of the SDEs at a single or a few time points. However, if a full sample path of the SDE has to be simulated or if the SDEs under consideration are part of a larger SDE system, then discretization schemes are typically more efficient.

\section{Pathwise Convergence Rates of the Euler Scheme and general ITÔ-TAYLOR METHODS}

The pathwise error criteria are very robust with respect to the global Lipschitz assumption. One of the simplest approximation schemes for equation (11) is the Euler scheme

$$
\bar{X}_{t_{k+1}}=\bar{X}_{t_{k}}+a\left(\bar{X}_{t_{k}}\right) \Delta+\sum_{j=1}^{m} b_{j}\left(\bar{X}_{t_{k}}\right) \Delta_{k} W^{(j)}, \quad k=0,1, \ldots,
$$

with $\bar{X}_{0}=x_{0}$, where $\Delta=T / n, t_{k}=k \Delta$ and $\Delta_{k} W=W_{t_{k+1}}-W_{t_{k}}$. The Euler scheme (and all other approximation methods that will be introduced below) depend on the stepsize $\Delta>0$, hence on $n \in \mathbb{N}$, but this dependence will be omitted whenever it is clear from the context.

From the results of Gyöngy [23] it follows that the Euler scheme has pathwise convergence order $1 / 2-\varepsilon$ also if the SDE coefficients are only locally Lipschitz continuous: for all $\varepsilon>0$

$$
\sup _{k=0, \ldots, n}\left|X_{t_{k}}-\bar{X}_{t_{k}}\right| \leq \eta_{\varepsilon}^{E} \cdot n^{-1 / 2+\varepsilon}
$$


almost surely for a finite and non-negative random variable $\eta_{\varepsilon}^{E}$ under the assumption that for all $N \in \mathbb{N}$ there exist constants $L_{N}>0$ such that

$$
|a(x)-a(y)|+\sum_{j=1}^{m}\left|b_{j}(x)-b_{j}(y)\right| \leq L_{N} \cdot|x-y|, \quad|x|,|y| \leq N .
$$

Thus, the pathwise convergence rate of the Euler scheme coincides up to an arbitrarily small $\varepsilon>0$ with its strong convergence rate $1 / 2$, but for the pathwise convergence rate no global Lipschitz assumption is required.

Jentzen, Kloeden \& Neuenkirch [37] observed that this is not a specific feature of the Euler scheme but, in fact, holds for general Itô-Taylor schemes of order $\gamma=0.5,1.0,1.5, \ldots$. For the definition of these schemes, see e.g. [39]. The Euler scheme corresponds to $\gamma=0.5$, while $\gamma=1.0$ yields the Milstein scheme

$$
\bar{X}_{t_{k+1}}=\bar{X}_{t_{k}}+a\left(\bar{X}_{t_{k}}\right) \Delta+\sum_{j=1}^{m} b_{j}\left(\bar{X}_{t_{k}}\right) \Delta_{k} W^{(j)}+\sum_{j_{1}, j_{2}=1}^{m} L^{j_{1}} b_{j_{2}}\left(\bar{X}_{t_{k}}\right) I_{j_{1}, j_{2}}\left(t_{k}, t_{k+1}\right)
$$

with the differential operators

$$
L^{j}=\sum_{k=1}^{d} b_{j}^{(k)} \frac{\partial}{\partial x^{k}}, \quad j=1, \ldots, m
$$

and the iterated Itô-integrals

$$
I_{j_{1}, j_{2}}(s, t)=\int_{s}^{t} \int_{s}^{\tau_{2}} d W_{\tau_{1}}^{\left(j_{1}\right)} d W_{\tau_{2}}^{\left(j_{2}\right)}, \quad j_{1}, j_{2}=1, \ldots, m
$$

The Itô-Taylor scheme of order 1.5 is usually called the Wagner-Platen scheme.

Theorem 2.1. Let $\gamma=0.5,1.0,1.5, \ldots$ Assume that $a, b_{1}, \ldots, b_{m} \in C^{2 \gamma+1}\left(\mathbb{R}^{d} ; \mathbb{R}^{d}\right)$ and moreover let $\bar{X}^{\gamma, n}$ be the Itô-Taylor scheme of order $\gamma$ with stepsize $\Delta=T / n$. Then for every $\varepsilon>0$ there exists a non-negative random variable $\eta_{\varepsilon}^{\gamma}$ such that

$$
\sup _{k=0, \ldots, n}\left|X_{t_{k}}(\omega)-\bar{X}_{t_{k}}^{\gamma, n}(\omega)\right| \leq \eta_{\varepsilon}^{\gamma}(\omega) \cdot n^{-\gamma+\varepsilon}
$$

for almost all $\omega \in \Omega$.

The main ingredients to obtain this result are the Burkholder-Davis-Gundy inequality, which implies that all moments of an Itô-integral are equivalent, the following BorelCantelli-type Lemma, and a localization procedure.

Lemma 2.2. (see [38]) Let $\alpha>0, c_{p} \geq 0$ for $p \geq 1$ and let $\left(Z_{n}\right)_{n \in \mathbb{N}}$ be a sequence of random variables with

$$
\left(\mathbf{E}\left|Z_{n}\right|^{p}\right)^{1 / p} \leq c_{p} \cdot n^{-\alpha}
$$

for all $p \geq 1$ and $n \in \mathbb{N}$. Then for every $\varepsilon>0$ there exists a finite and non-negative random variable $\eta_{\varepsilon}$ such that

$$
\left|Z_{n}\right| \leq \eta_{\varepsilon} \cdot n^{-\alpha+\varepsilon}
$$

almost surely for all $n \in \mathbb{N}$. 
The Burkholder-Davis-Gundy inequality and the Borel-Cantelli-type Lemma allow one to show that the Itô-Taylor scheme of order $\gamma$ has pathwise convergence rate $\gamma-\varepsilon$ for smooth and bounded coefficients with bounded derivatives, thereby extending the classical mean-square convergence analysis in 39. Then a localization argument is applied to avoid the boundedness assumptions. Roughly speaking, this localization argument works as follows: A fixed sample path $X_{t}(\omega), t \in[0, T]$, of the SDE solution is bounded, i.e. stays in some open set $B(\omega)$. However for the SDE

$$
d Y_{t}=\widetilde{a}\left(Y_{t}\right) d t+\sum_{j=1}^{m} \widetilde{b}_{j}\left(Y_{t}\right) d W_{t}^{(j)}, \quad Y_{0}=x_{0}
$$

with smooth and bounded coefficients $\widetilde{a}, \widetilde{b}_{j}$ with bounded derivatives, which coincide with the ones of the original SDE on $B(\omega)$, the solution sample path $Y_{t}(\omega), t \in[0, T]$, coincides with $X_{t}(\omega), t \in[0, T]$. Asymptotically this also holds for the corresponding sample paths of the $\gamma$-Itô-Taylor schemes, so the pathwise convergence rates carry over.

Note that all the examples of SDEs given in the introduction take non-negative values only, so good approximation schemes should preserve this structural property. The (explicit) Euler scheme is, in general, not such a scheme, since its increments are conditionally Gaussian. For example, in case of the CIR process

$$
d X_{t}=\kappa\left(\lambda-X_{t}\right) d t+\theta \sqrt{X_{t}} d W_{t}, \quad X_{0}=x_{0}>0
$$

the transition density of the Euler scheme reads as

$$
p(y ; x)=\frac{1}{\sqrt{2 \pi \theta^{2} x \Delta}} \exp \left(-\frac{\left(y-(x+\kappa(\lambda-x) \Delta)^{2}\right.}{2 \theta^{2} x \Delta}\right), \quad y \in \mathbb{R}, x>0,
$$

so negative values can be obtained with positive probability even in the first step. This has lead to many ad-hoc corrections to prevent termination of the Euler scheme. The truncated Euler scheme

$$
\bar{X}_{t_{k+1}}=\bar{X}_{t_{k}}+\kappa\left(\lambda-\bar{X}_{t_{k}}\right) \Delta+\theta \sqrt{\bar{X}_{t_{k}}^{+}} \Delta_{k} W, \quad k=0,1, \ldots
$$

was proposed in [14], while the scheme

$$
\bar{X}_{t_{k+1}}=\bar{X}_{t_{k}}+\kappa\left(\lambda-\bar{X}_{t_{k}}\right) \Delta+\theta \sqrt{\left|\bar{X}_{t_{k}}\right|} \Delta_{k} W, \quad k=0,1, \ldots
$$

was studied in [28]. Both approaches extend the mapping $[0, \infty) \ni x \mapsto \sqrt{x} \in[0, \infty)$ suitably to negative values of $x$. For the CIR process this idea was taken further by Lord, Koekkoek \& van Dijk [40], who also proposed modifications of the drift coefficient for negative values of the state space.

Example 2.3. The following table shows the average number of negative steps per path for the above Euler approximations of the CIR process. Scenario I (taken from [2]), corresponds to the parameters

$$
x_{0}=0.05, \quad \kappa=5.07, \quad \lambda=0.0457, \quad \theta=0.48, \quad T=5
$$

while Scenario II (taken from [11]) uses

$$
x_{0}=0.09, \quad \kappa=2, \quad \lambda=0.09, \quad \theta=1, \quad T=5 .
$$


The stepsize for the Euler schemes is given by $\Delta=T / n$ with $n=512$.

\begin{tabular}{c||c|c} 
average negative steps of / for & Scenario I & Scenario II \\
\hline \hline Euler scheme (2) & 0.9141 & 64.8611 \\
\hline Euler scheme (3) & 1.0590 & 74.5017
\end{tabular}

The empirical frequency of negative paths is 0.4913 in Scenario I and 0.9990 in Scenario II. These results were obtained by a Monte Carlo simulation with $N=10^{6}$ repetition. They clearly indicate that the Euler scheme (3) has a tendency for negative "excursions". This can also be seen in Figure1, which shows a sample path of the (linearly interpolated) Euler schemes (2) and (3) using the same path of the driving Brownian motion. The parameters used in this figure correspond to Scenario II.

For general SDEs the procedure of modifying the coefficients outside the support of the solution has been introduced systematically in [37. For an SDE

$$
d X_{t}=a\left(X_{t}\right) d t+\sum_{j=1}^{m} b_{j}\left(X_{t}\right) d W_{t}^{(j)}, \quad X_{0}=x_{0}
$$

which takes values in a domain $D \subset \mathbb{R}^{d}$, i.e.

$$
\mathbf{P}\left(X_{t} \in D, t \geq 0\right)=1,
$$

the auxiliary coefficients

$$
\begin{array}{ll}
\widetilde{a}(x)=a(x) \cdot \mathbf{1}_{D}(x)+f(x) \cdot \mathbf{1}_{E}(x), & x \in \mathbb{R}^{d} \\
\widetilde{b}_{j}(x)=b_{j}(x) \cdot \mathbf{1}_{D}(x)+g_{j}(x) \cdot \mathbf{1}_{E}(x), & x \in \mathbb{R}^{d}, \quad j=1, \ldots, m
\end{array}
$$

with $E=\mathbb{R}^{d} \backslash D$ are introduced there. A modified Itô-Taylor scheme of order $\gamma$ based on the auxiliary functions $f$ and $g$ is then the corresponding standard Itô-Taylor scheme for the SDE

$$
d X_{t}=\widetilde{a}\left(X_{t}\right) d t+\sum_{j=1}^{m} \widetilde{b}_{j}\left(X_{t}\right) d W_{t}^{(j)}
$$

with a suitable definition of the derivatives of the coefficients on $\partial D$, see [37] for details. This method is well-defined as long as the coefficients of the equation are $(2 \gamma+1)$-times differentiable on $D$ and the auxiliary functions are $(2 \gamma-1)$-times differentiable on $E$. The purpose of the auxiliary functions is twofold: to obtain a well-defined approximation scheme and to bring the numerical scheme back to $D$ if it leaves $D$. In particular, the auxiliary functions can always be chosen to be affine or even constant. It was shown by Jentzen, Kloeden \& Neuenkirch [37] that Theorem 2.1 adapts to modified Itô-Taylor schemes for SDEs on domains $D \subset \mathbb{R}^{d}$.

Theorem 2.4. Let $X$ be the solution of SDE (4) satisfying condition (5). Moreover let $\gamma=0.5,1.0,1.5, \ldots$ and assume that

$$
a \in C^{2 \gamma+1}\left(D ; \mathbb{R}^{d}\right), \quad b \in C^{2 \gamma+1}\left(D ; \mathbb{R}^{d, m}\right)
$$



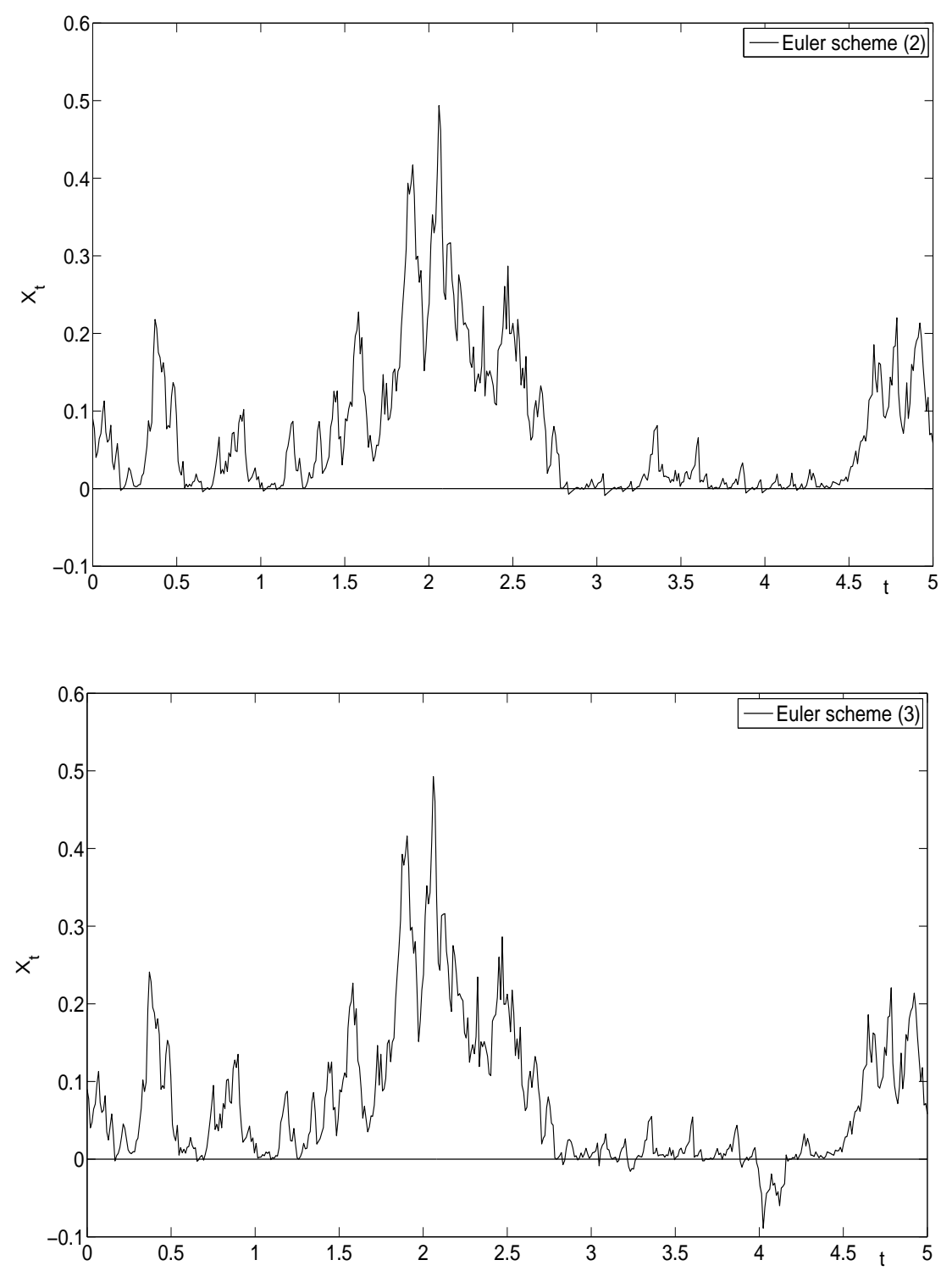

Figure 1. A path of Euler scheme (2) vs. Euler scheme (3) for the CIR process and Scenario II

and

$$
f \in C^{2 \gamma-1}\left(E ; \mathbb{R}^{d}\right), \quad g \in C^{2 \gamma-1}\left(E ; \mathbb{R}^{d, m}\right) .
$$

Finally let $\widetilde{X}^{\gamma, n}$ be the modified Itô-Taylor method for $X$ based on the auxiliary functions $f$ and $g$ with stepsize $\Delta=T / n$. Then for every $\varepsilon>0$ there exists a finite and nonnegative random variable $\eta_{\gamma, \varepsilon}^{f, g}$ such that

$$
\sup _{k=0, \ldots, n}\left|X_{t_{k}}(\omega)-\tilde{X}_{t_{k}}^{\gamma, n}(\omega)\right| \leq \eta_{\gamma, \varepsilon}^{f, g}(\omega) \cdot n^{-\gamma+\varepsilon}
$$

for almost all $\omega \in \Omega$ and all $n \in \mathbb{N}$. 
In the case of the Euler scheme, i.e. $\gamma=0.5$, the assumptions on $a$ and $b$ can be weakened to the assumption that $a$ and $b$ are locally Lipschitz continuous on $D$. For SDEs on domains in mathematical finance this condition is typically satisfied. In fact, in most cases the coefficients are infinitely differentiable.

The CIR process satisfies

$$
\mathbf{P}\left(X_{t}>0 \text { for all } t \geq 0\right)=1
$$

if and only if $2 \kappa \lambda \geq \theta^{2}$. The latter assumption is typically satisfied in interest rate applications of the CIR process. Hence, modified Taylor schemes can be used here with $D=(0, \infty)$. The truncated Euler scheme (2) corresponds to the auxiliary functions $f(x)=a(x), g(x)=0, x \leq 0$, while the scheme (3) uses the auxiliary functions $f(x)=$ $a(x), g(x)=\sqrt{-x}, x \leq 0$. Note that $2 \kappa \lambda \geq \theta^{2}$ is satisfied in Scenario I, but not in Scenario II.

For structure preserving integration of the CIR process also the symmetrized Euler method

$$
\widetilde{X}_{t_{k+1}}=\left|\widetilde{X}_{t_{k}}+\kappa\left(\lambda-\widetilde{X} t_{k}\right) \Delta+\theta \sqrt{\widetilde{X}_{t_{k}}} \Delta_{k} W\right|, \quad k=0,1, \ldots
$$

was proposed in [10, 8]. While the modified Euler schemes (2) and (3) may leave $(0, \infty)$ and are then forced back in the next steps, this scheme is always non-negative. Adapting this to general SDEs, which take values in a domain $D$, leads to the reflected Euler schemes, see e.g. [44, which are given by

$$
\widetilde{X}_{t_{k+1}}^{\psi}=H_{t_{k+1}}^{\psi} \cdot \mathbf{1}_{D}\left(H_{t_{k+1}}^{\psi}\right)+\psi\left(H_{t_{k+1}}^{\psi}\right) \cdot \mathbf{1}_{\mathbb{R}^{d} \backslash D}\left(H_{t_{k+1}}^{\psi}\right)
$$

with $\tilde{X}_{0}^{\psi}=x_{0}$, where

$$
H_{t_{k+1}}^{\psi}=\widetilde{X}_{t_{k}}^{\psi}+a\left(\widetilde{X}_{t_{k}}^{\psi}\right) \Delta+\sum_{j=1}^{m} b_{j}\left(\widetilde{X}_{t_{k}}^{\psi}\right) \Delta_{k} W^{(j)}
$$

and a measurable projection function $\psi: \mathbb{R}^{d} \backslash D \rightarrow D \cup \partial D$. A straightforward modification of the above theorem yields a pathwise convergence order $1 / 2-\varepsilon$ for these reflected Euler schemes if the SDE coefficients are twice continuously differentiable on $D$. In the same way reflected Itô-Taylor schemes of arbitrary order can be constructed and analyzed.

The symmetrized Euler scheme (6) corresponds to the reflection function $\psi(x)=|x|$. The results on modified Itô-Taylor schemes and reflected Euler methods apply also to the generalized Ait-Sahalia model with $D=(0, \infty)$ if $r>1, \rho<(1+r) / 2$, to the Heston model with $D=(0, \infty)^{2}$ if $2 \kappa \lambda \geq \theta^{2}$ and to the $3 / 2$-model with $D=(0, \infty)^{2}$ and no further restrictions on the parameter.

Example 2.5. To illustrate the above results consider Scenario I for the Cox-IngersollRoss process. Figure 2 shows for two different sample paths $\omega \in \Omega$ the maximum error in the discretization points, i.e.

$$
\sup _{k=0, \ldots, n}\left|X_{t_{k}}(\omega)-\bar{X}_{t_{k}}(\omega)\right|
$$

of

(i) the truncated Euler scheme (2) 
(ii) the symmetrized Euler scheme (6)

(iii) the modified Milstein scheme with auxiliary functions $f(x)=\kappa(\lambda-x), g(x)=0$, i.e. a truncated Milstein scheme.

To estimate the pathwise maximum error for the above approximation schemes the CoxIngersoll-Ross process have been discretized with a very small step size using scheme (2). In Figure 2 log-log-coordinates are used, so the dots indicate the convergence orders 0.5
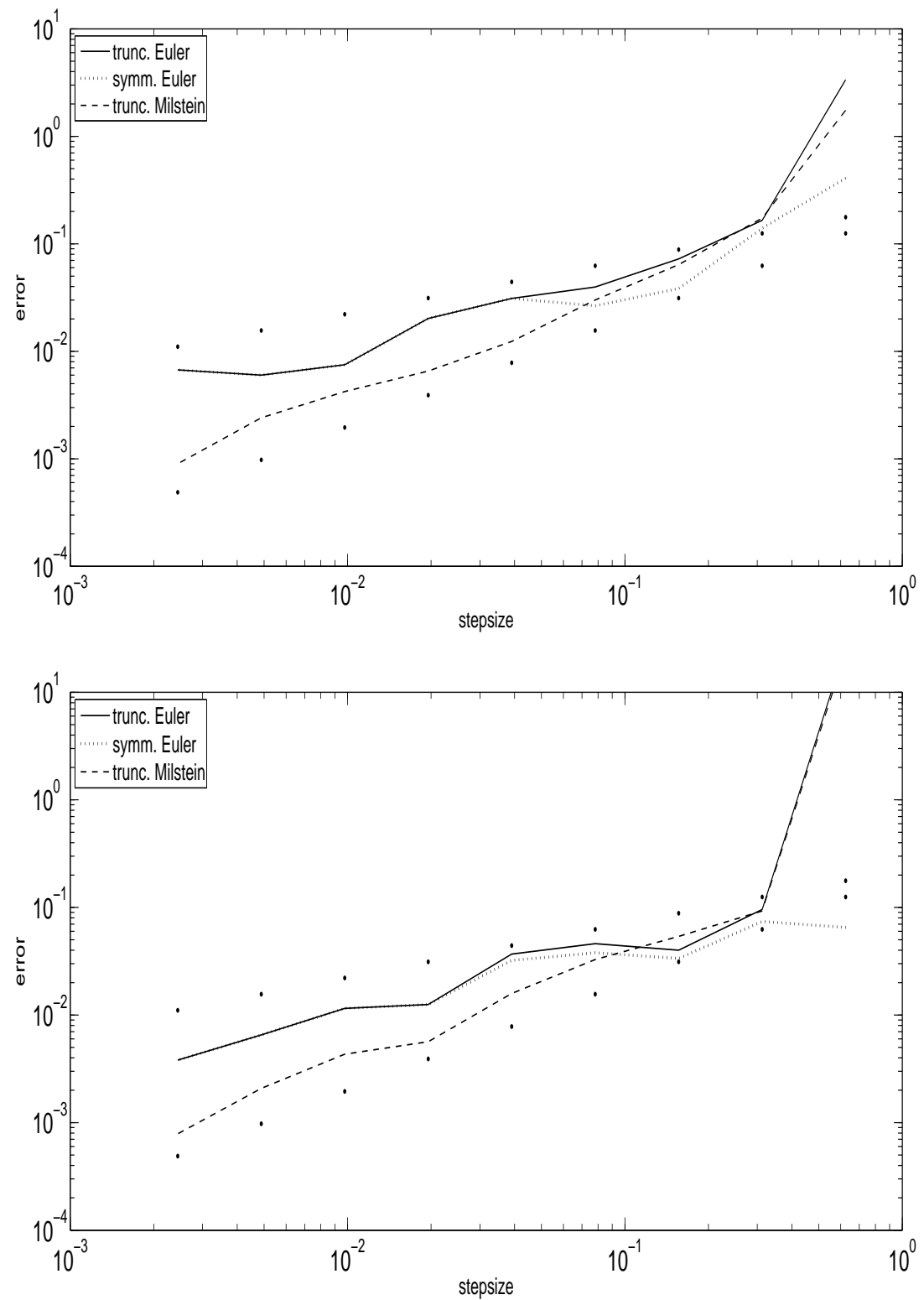

Figure 2. Pathwise maximum error vs. step size for two sample paths for the Cox-Ingersoll-Ross model for Scenario I

and 1. The pathwise convergence rates of all three approximation schemes are in good accordance with the theoretically predicted rates for moderate and small step sizes. For small step sizes both Euler schemes do not take negative values and hence coincide. 
Moreover, for small step sizes the Milstein scheme is superior due to its first order convergence.

Numerical methods with pathwise convergence rates of high order are thus available also for SDEs with non-globally Lipschitz coefficients. However, while pathwise convergence rates are very important for the analysis of random dynamical systems [7, 17, one of the main objectives in mathematical finance is the pricing of (pathdependent) European-type derivatives, which means to compute real numbers $\mathbf{E} \Phi(X)$ where $\Phi: C\left([0, T] ; \mathbb{R}^{d}\right) \rightarrow \mathbb{R}$ is the discounted payoff of the derivative. Since the integrability of the random constants in the error bounds is an open problem, the above pathwise convergence rates do not imply weak or strong convergence rates. Nevertheless, if $\Phi$ is bounded and continuous and if $\bar{X}^{\gamma}=\left(\bar{X}_{t}^{\gamma}\right)_{t \in[0, T]}$ is the piecewise linear interpolation of the $\gamma$-Itô-Taylor scheme (standard, modified or reflected) then

$$
\mathrm{E} \Phi\left(\bar{X}^{\gamma}\right) \longrightarrow \mathrm{E} \Phi(X)
$$

for $n \rightarrow \infty$, so for bounded and continuous pay-offs (e.g. put options) one obtains at least the convergence of the corresponding standard Monte Carlo estimators for the option price. The same is true for barrier options with payoff of the form

$$
\Phi(X)=\phi\left(X_{T}\right) \mathbf{1}_{\left\{K_{1} \leq\left|X_{t}\right| \leq K_{2}, t \in[0, T]\right\}}
$$

with $0 \leq K_{1} \leq K_{2}<\infty$, if $\phi$ is bounded and continuous and the law of $\sup _{t \in[0, T]}\left|X_{t}\right|$ and $\inf _{t \in[0, T]}\left|X_{t}\right|$ has a density with respect to the Lebesgue measure.

\section{The Explicit Euler Scheme: Criteria for Weak and Strong Convergence}

It was shown by Higham, Mao \& Stuart in [29] that the explicit Euler scheme

$$
\begin{aligned}
\bar{X}_{t_{k+1}} & =\bar{X}_{t_{k}}+a\left(\bar{X}_{t_{k}}\right) \Delta+\sum_{j=1}^{m} b_{j}\left(\bar{X}_{t_{k}}\right) \Delta_{k} W^{(j)}, \quad k=0,1, \ldots, \\
\bar{X}_{0} & =x_{0}
\end{aligned}
$$

is strongly convergent if the coefficients are locally Lipschitz continuous on $\mathbb{R}^{d}$ and a moment condition for the SDE and its Euler approximation is satisfied. This result can be extended to SDE on domains and the modified or reflected Euler scheme.

Theorem 3.1. Let $X$ be the solution of SDE (4) satisfying condition (5). Moreover, let $\widetilde{X}^{n}$ be the modified Euler scheme based on the auxiliary functions $f \in C\left(E ; \mathbb{R}^{d}\right)$, $g \in C\left(E ; \mathbb{R}^{d, m}\right)$ with stepsize $\Delta=T / n$ or let $\tilde{X}^{n}$ be the reflected Euler scheme based on the projection function $\psi: E \rightarrow D \cup \partial D$ with stepsize $\Delta=T / n$. Assume that

$$
a \in C^{2}\left(D ; \mathbb{R}^{d}\right), \quad b \in C^{2}\left(D ; \mathbb{R}^{d, m}\right)
$$

and furthermore, assume that for some $p>2$

$$
\sup _{n \in \mathbb{N}} \mathbf{E} \max _{k=0, \ldots n}\left|\tilde{X}_{t_{k}}^{n}\right|^{p}+\mathbf{E} \sup _{t \in[0, T]}\left|X_{t}\right|^{p}<\infty .
$$


Then

$$
\lim _{n \rightarrow \infty} \mathbf{E} \max _{k=0, \ldots, n}\left|X_{t_{k}}-\widetilde{X}_{t_{k}}^{n}\right|^{2}=0
$$

Proof. From the results of the previous section

$$
\lim _{n \rightarrow \infty} \max _{k=0, \ldots, n}\left|X_{t_{k}}-\tilde{X}_{t_{k}}^{n}\right|=0
$$

hold, almost surely. However, assumption (7) implies the uniform integrability of

$$
\max _{k=0, \ldots n}\left|X_{t_{k}}-\tilde{X}_{t_{k}}^{n}\right|^{2}, \quad n \in \mathbb{N} .
$$

The assertion now follows, since uniform integrability allows integration to the limit.

Note that assumption (7) is easily verified if the SDE coefficients have linear growth on $D$, i.e.

$$
|a(x)|+\sum_{j=1}^{m}\left|b_{j}(x)\right| \leq C \cdot(1+|x|), \quad x \in D,
$$

for some $C>0$. Turning back to the Cox-Ingersoll-Ross process this gives us strong convergence of the Euler schemes (2), (3) and (6) under the assumption $2 \kappa \lambda \geq \theta^{2}$. Note that for the Euler schemes (2) and (3) strong convergence without a restriction on the parameter has been shown in [14] and [28] using a Yamada function technique. This technique has also been applied by Gyöngy \& Rásonyi in [24] to obtain the following result:

Theorem 3.2. Let $a_{1}, a_{2}, b: \mathbb{R} \rightarrow \mathbb{R}$. Consider the one-dimensional SDE

$$
d X_{t}=\left(a_{1}\left(X_{t}\right)+a_{2}\left(X_{t}\right)\right) d t+b\left(X_{t}\right) d W_{t}, \quad t \in[0, T], \quad X_{0}=x_{0} \in \mathbb{R}
$$

and let $\bar{X}^{n}$ be the corresponding Euler scheme with stepsize $\Delta=T / n$. Moreover, let $a_{2}$ be monotonically decreasing and assume that there exists constants $\alpha \in[0,1 / 2], \beta \in(0,1]$ and $C>0$ such that

$$
\begin{gathered}
\left|a_{1}(x)-a_{1}(y)\right| \leq C \cdot|x-y|, \quad\left|a_{2}(x)-a_{2}(y)\right| \leq C \cdot|x-y|^{\beta}, \\
|b(x)-b(y)| \leq C \cdot|x-y|^{\frac{1}{2}+\alpha}
\end{gathered}
$$

for all $x, y \in \mathbb{R}$. Then, for all $p \in \mathbb{N}$, there exist constants $K_{p}^{\alpha, \beta}>0$ such that

$$
\mathbf{E} \max _{k=0, \ldots, n}\left|X_{t_{k}}-\bar{X}_{t_{k}}^{n}\right|^{p} \leq \begin{cases}K_{p}^{0, \beta} \cdot \frac{1}{\log (n)} & \text { for } \alpha=0 \\ K_{p}^{\alpha, \beta} \cdot\left(\frac{1}{n^{\alpha}}+\frac{1}{n^{\beta / 2}}\right) & \text { for } \alpha \in(0,1 / 2) \\ K_{p}^{\alpha, \beta} \cdot\left(\frac{1}{n^{p / 2}}+\frac{1}{n^{\beta p / 2}}\right) & \text { for } \alpha=1 / 2\end{cases}
$$

This result can be applied to the CEV model

$$
d X_{t}=\mu X_{t} d t+\sigma X_{t}^{\gamma} d W_{t}
$$

if the mapping $[0, \infty) \ni x \mapsto x^{\gamma} \in[0, \infty)$ is extended to $(-\infty, 0)$, e.g. as $\left(x^{+}\right)^{\gamma}$ or $|x|^{\gamma}$. Theorem 3.2 then yields strong convergence of the corresponding Euler schemes. 
Example 3.3. Whether the convergence rates predicted from Theorem 3.2 are sharp for the CEV model remains an open problem. The following simulation study suggests that the Euler scheme has strong convergence order $1 / 2$, at least for some parameter constellations. To better preserve the positivity of the CEV process, the Euler scheme is applied to the SDE

$$
d X_{t}=\mu\left|X_{t}\right| d t+\sigma\left(X_{t}^{+}\right)^{\gamma} d W_{t}
$$

which still fulfills the assumptions of Theorem 3.2 with $a_{2}=0$, i.e. $\beta=1$, and $\alpha=$ $\gamma-1 / 2$. Its solution coincides with the CEV process.

Figure 3 shows the empirical root mean square maximum error in the discretization points versus the step size for the parameters

$$
\begin{aligned}
& \text { Set I: } \quad \mu=0.1, \quad \sigma=0.3, \quad \gamma=0.75, \quad T=1, \quad x_{0}=0.2 \\
& \text { Set II: } \quad \mu=0.2, \quad \sigma=0.5, \quad \gamma=0.55, \quad T=1, \quad x_{0}=0.5
\end{aligned}
$$

The empirical mean square maximum error in the discretization points is estimated by

$$
\left(\frac{1}{N} \sum_{i=1}^{N} \max _{k=0, \ldots, n}\left|X_{t_{k}}^{*,(i)}-\bar{X}_{t_{k}}^{n,(i)}\right|^{2}\right)^{1 / 2}
$$

with $N=5 \cdot 10^{4}$. Here $X^{*}$ is the numerical reference solution obtained by using the same Euler scheme with very small step size and $X^{*,(i)}, \bar{X}^{n,(i)}$ are independent copies of $X^{*}, \bar{X}^{n}$. For both sets of parameter a good accordance with the convergence order $1 / 2$ is obtained. (The dots in the figure indicate convergence order 1/2). A regression of

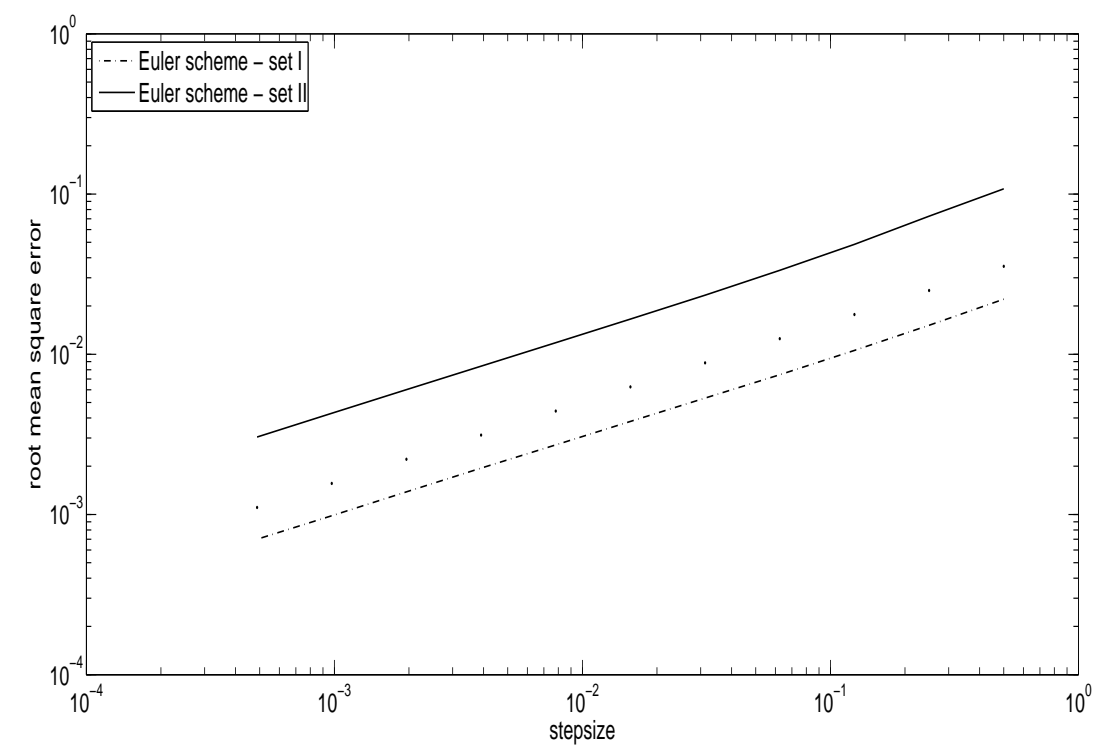

Figure 3. Root mean square error of the Euler scheme vs. step size for the CEV process for the parameter sets I and II

the numerical data yields moreover the empirical convergence order 0.493923 for set I, respectively 0.509903 for set II. 
But do Theorems 3.1 and 3.2 have any consequences for the other examples? Unfortunately not: for the Heston, Ait-Sahalia and 3/2-models, no linear growth condition is satisfied. Even worse, for the Ait-Sahalia model and the 3/2-model the moments of the Euler scheme explode! In the case of the 3/2-model the latter can be deduced from the following Theorem, which was obtained by Hutzenthaler, Jentzen \& Kloeden in 34.

Theorem 3.4. Let $a, b: \mathbb{R} \rightarrow \mathbb{R}$ and assume that the one-dimensional $S D E$

$$
d X_{t}=a\left(X_{t}\right) d t+b\left(X_{t}\right) d W_{t}, \quad t \in[0, T], \quad X_{0}=x_{0} \in \mathbb{R}
$$

has a unique strong solution with

$$
\sup _{t \in[0, T]} \mathbb{E}\left|X_{t}\right|^{p}<\infty
$$

for one $p \in[1, \infty)$. Moreover, let $b\left(x_{0}\right) \neq 0$ and let $C \geq 1, \beta>\alpha>1$ be constants such that

$$
\max (|a(x)|,|b(x)|) \geq \frac{1}{C} \cdot|x|^{\beta} \quad \text { and } \min (|a(x)|,|b(x)|) \leq C \cdot|x|^{\alpha}
$$

for all $|x| \geq C$. Then, the corresponding Euler scheme $\bar{X}^{n}$ with stepsize $\Delta=T / n$ satisfies

$$
\lim _{n \rightarrow \infty} \mathbb{E}\left|X_{T}-\bar{X}_{T}^{n}\right|^{p}=\infty \quad \text { and }\left.\quad \lim _{n \rightarrow \infty}|\mathbb{E}| X_{T}\right|^{p}-\mathbb{E}\left|\bar{X}_{T}^{n}\right|^{p} \mid=\infty
$$

In the case of the 3/2-model, which has finite moments up to order $p<2+\frac{2 c_{1}}{c_{3}^{2}}$, the coefficients are

$$
a(x)=-c_{2} x^{2}+c_{1} c_{2} x, \quad b(x)=c_{3}\left(x^{+}\right)^{3 / 2}, \quad x \in \mathbb{R},
$$

so the assumptions of the above Theorem are satisfied for $\alpha=3 / 2, \beta=2$ and $C$ sufficiently large.

Concerning the Ait-Sahalia model, the moments of the Euler scheme already explode in the second step. Here the first step of the Euler scheme has a Gaussian distribution with mean $x_{0}+\left(\alpha_{-1} x_{0}^{-1}-\alpha_{0}+\alpha_{1} x_{0}-\alpha_{2} x_{0}^{r}\right) \Delta$ and variance $\alpha_{3}^{2} x_{0}^{2 \rho} \Delta$. The inverse of the first step must be computed for the second step of the Euler scheme, so the moments of the second step are infinite, since inverse moments of a Gaussian random variable do not exist.

Why the moments of the Euler scheme diverge for superlinearly growing coefficients - even without a singularity - can be nicely illustrated by considering the SDE

$$
d X_{t}=-X_{t}^{3} d t+\sigma d W_{t}, \quad X_{0}=x_{0}
$$

with $\sigma \geq 0$ for which the Euler scheme reads as

$$
\bar{X}_{t_{k+1}}^{n}=\bar{X}_{t_{k}}^{n}\left(1-\left|\bar{X}_{t_{k}}^{n}\right|^{2} \Delta\right)+\sigma \Delta_{k} W .
$$

In the deterministic case, i.e., (9) and (10) with $\sigma=0$, the Euler approximation of the deterministic equation is known to be unstable if the initial value is large (see e.g. Chapter 6 in [16]). For example, if $x_{0}=n, T=1$ and $\Delta=n^{-1}$ then

$$
\bar{X}_{t_{1}}^{n}=n\left(1-\frac{n^{2}}{n}\right) \approx-n^{2}
$$

and therefore

$$
\bar{X}_{t_{2}}^{n}=\bar{X}_{t_{1}}^{n}\left(1-\left|\bar{X}_{t_{1}}^{n}\right|^{2} \Delta\right) \approx n^{5}
$$


Iterating this further, one obtains

$$
\left|\bar{X}_{t_{k}}^{n}\right| \gtrsim n^{\left(2^{k}\right)}
$$

for $k=0,1, \ldots, n$. Thus, $\bar{X}_{t_{n}}^{n}$ grows double-exponentially fast in $n$. In the presence of noise $(\sigma>0)$ there is an exponentially small event that the Brownian motion leaves the interval $[-2 n, 2 n]$ and on this event the approximations grow double-exponentially fast due to the deterministic dynamics. Consequently this double-exponentially growth can not be compensated by the exponentially small probability of this event, which leads to the moment explosion of the Euler approximation.

Example 3.5. That rare events lead to the explosion of the moments of the Euler scheme can be also seen from the following numerical example. Consider the volatility process in the $3 / 2$-model

$$
d V_{t}=c_{1} V_{t}\left(c_{2}-V_{t}\right) d t+c_{3} V_{t}^{3 / 2} d W_{t}, \quad V_{0}=v_{0}>0
$$

with

$$
c_{1}=1.2, \quad c_{2}=0.8, \quad c_{3}=1, \quad T=4, \quad v_{0}=0.5
$$

and try to compute

$$
\mathbf{E}\left|X_{T}\right|=0.566217
$$

using the standard Monte Carlo estimator

$$
\frac{1}{N} \sum_{i=1}^{N}\left|\bar{X}_{T}^{n,(i)}\right|
$$

where $\bar{X}_{T}^{n,(1)}, \ldots, \bar{X}_{T}^{n,(N)}$ are iid copies of $\bar{X}_{T}^{n}$. The exact value for $\mathbf{E}\left|X_{T}\right|$ is computed using the inverse moments of the CIR process, see e.g. [31]. While for a moderate number of repetitions the estimator seems to converge for small step sizes (and the 'Inf'-outputs seem to be some numerical instabilities due to the large step sizes), the estimator explodes even for small step sizes when increasing the number of repetitions - as predicted by Theorem 3.4. Despite of this the Euler scheme for this SDE converges pathwise with rate $1 / 2-\varepsilon$ due to Theorem 2.4 .

\begin{tabular}{c||c|c|c|c|c|c|} 
Repetitions $N /$ stepsize $\Delta$ & $2^{0}$ & $2^{-2}$ & $2^{-4}$ & $2^{-6}$ & $2^{-8}$ & $2^{-10}$ \\
\hline \hline $10^{3}$ & 6.327232 & Inf & Inf & 0.550185 & 0.553499 & 0.555069 \\
\hline $10^{4}$ & 6.894698 & Inf & Inf & Inf & 0.562716 & 0.563352 \\
\hline $10^{5}$ & 7.430606 & Inf & Inf & Inf & 0.566218 & 0.567106 \\
\hline $10^{6}$ & 7.227379 & Inf & Inf & Inf & Inf & 0.565750 \\
\hline $10^{7}$ & 7.279187 & Inf & Inf & Inf & Inf & Inf
\end{tabular}

A similar moment explosion arises if a Multi-level Monte Carlo method is used to estimate $\mathbf{E}\left|X_{T}\right|$. This is shown, also for more general SDEs, in [36].

However, in some cases using the Euler scheme one still obtains a convergent Monte Carlo estimator for functionals of the type $\mathbf{E} \phi\left(X_{T}\right)$. The standard Euler-based estimator 
for the latter quantity is

$$
\frac{1}{N} \sum_{i=1}^{N} \phi\left(\bar{X}_{T}^{n,(i)}\right)
$$

In the classical case, i.e. if $a, b, \phi \in C^{4}(\mathbb{R} ; \mathbb{R})$ with at most polynomially growing derivatives and $a, b$ globally Lipschitz, one has

$$
\mathbf{E}\left|\frac{1}{N} \sum_{i=1}^{N} \phi\left(\bar{X}_{T}^{n,(i)}\right)-\mathbf{E} \phi\left(X_{T}\right)\right|^{2} \leq K_{\text {Bias }} \cdot \frac{1}{n^{2}}+K_{M C} \cdot \frac{1}{N},
$$

see e.g. 39]. The first term on the right hand side corresponds to the squared bias of the Euler scheme, while the second term corresponds to the variance of the Monte Carlo simulation. It is thus optimal to choose $N=n^{2}$ for balancing both terms with respect to the computational cost (number of arithmetic operations, function evaluations and random numbers used), see [18]. The corresponding Monte Carlo estimator has then convergence order $1 / 3$ in terms of the computational cost.

Hutzenthaler \& Jentzen could show in [32] that if the global Lipschitz assumption on the drift-coefficient is weakened to

$$
(x-y)(a(x)-a(y)) \leq L(x-y)^{2}, \quad x, y \in \mathbb{R}
$$

for some $L>0$, then one still has

$$
\left|\frac{1}{N^{2}} \sum_{i=1}^{N^{2}} \phi\left(\bar{X}_{T}^{N,(i)}\right)-\mathbf{E} \phi\left(X_{T}\right)\right| \leq \eta_{\varepsilon} \cdot N^{-(1-\varepsilon)}
$$

almost surely for all $\varepsilon>0$ and almost-surely finite and non-negative random variables $\eta_{\varepsilon}$.

Weak approximation under non-standard assumptions is also studied by Milstein \& Tretyakov in [42]. In their approach, simulations which leave a ball with sufficiently large radius are discarded. In the context of the Euler scheme with equidistant stepsize this estimator reads as

$$
\frac{1}{N} \sum_{i=1}^{N} \phi\left(\bar{X}_{T}^{n,(i)}\right) \cdot \mathbf{1}_{\left\{\sup _{k=0, \ldots, n}\left|X_{t_{k}}^{n,(i)}\right| \leq R\right\}} .
$$

For coefficients $a, b$ and functions $\phi$ satisfying a Lyapunov-type condition still a convergent Monte Carlo estimator is obtained, when matching the discarding radius $R$ appropriately to the number of repetitions $N$ and the stepsize of the discretization $n$.

Condition (12) on the drift coefficient is the so-called one-sided Lipschitz condition. This condition is also very useful to obtain strong convergence results for implicit Euler methods and tamed Euler schemes, which will be explained in the next section. Very recently a unifying framework for the analysis of Euler-type methods has been provided in 33 . 


\section{Strong COnVERGence of implicit And tAmed Euler schemes}

The condition in Theorem 3.1 for the strong convergence of the Euler scheme which is usually difficult to verify is the finiteness of its moments, i.e.

$$
\sup _{n \in \mathbb{N}} \mathbf{E} \max _{k=0, \ldots, n}\left|\bar{X}_{t_{k}}^{n}\right|^{p}<\infty
$$

for some $p>2$. Moreover, this condition may even fail to hold for specific equations, see Theorem 3.4. However, both problems can be overcome in some situations if appropriate drift-implicit Euler schemes are used. The split-step backward Euler scheme is defined as

$$
X_{t_{k}}^{*}=\bar{X}_{t_{k}}+a\left(X_{t_{k}}^{*}\right) \Delta, \quad \bar{X}_{t_{k+1}}=X_{t_{k}}^{*}+\sum_{j=1}^{m} b_{j}\left(X_{t_{k}}^{*}\right) \Delta_{k} W^{(j)}
$$

for $k=0,1, \ldots$ with $\bar{X}_{0}=x_{0}$, while the backward or drift-implicit Euler scheme reads as

$$
\bar{X}_{t_{k+1}}=\bar{X}_{t_{k}}+a\left(\bar{X}_{t_{k+1}}\right) \Delta+\sum_{j=1}^{m} b_{j}\left(\bar{X}_{t_{k}}\right) \Delta_{k} W^{(j)} .
$$

Both schemes are defined via an implicit equation, whose solvability relies on the properties of the drift-coefficient $a$. The following result has been obtained by Higham, Mao \& Stuart in [29].

Theorem 4.1. Let $a, b_{j} \in C^{1}\left(\mathbb{R}^{d} ; \mathbb{R}^{d}\right), j=1, \ldots, m$, and assume that there exist constants $L_{1}, L_{2}>0$ such that

$$
\begin{array}{cc}
\langle x-y, a(x)-a(y)\rangle \leq L_{1} \cdot|x-y|^{2}, & x, y \in \mathbb{R}^{d}, \\
\sum_{j=1}^{m}\left|b_{j}(x)-b_{j}(y)\right|^{2} \leq L_{2} \cdot|x-y|^{2}, & x, y \in \mathbb{R}^{d} .
\end{array}
$$

Then, the split-step backward Euler scheme given by (13) with stepsize $\Delta=T / n$ is well defined for $\Delta<\Delta_{*}:=1 / \max \left\{1+2 L_{1}, 4 L_{2}\right\}$ and satisfies

$$
\lim _{n \rightarrow \infty} \mathbf{E} \max _{k=0, \ldots, n}\left|X_{t_{k}}-\bar{X}_{t_{k}}^{n}\right|^{2}=0 .
$$

The conditions on the coefficients imply that the SDE has bounded moments of any order, and also allow one to show that the split-step Euler method has moments of any order. The implicitness of the method is crucial for the latter. Furthermore, the split-step Euler method coincides with the explicit Euler method for the perturbed SDE

$$
d X_{t}^{\Delta}=a\left(h_{\Delta}\left(X_{t}^{\Delta}\right)\right) d t+\sum_{j=1}^{m} b_{j}\left(h_{\Delta}\left(X_{t}^{\Delta}\right)\right) d W^{(j)}(t), \quad X_{0}^{\Delta}=x_{0} .
$$

Here the function $h_{\Delta}: \mathbb{R}^{d} \rightarrow \mathbb{R}^{d}$ is defined as the unique solution of the equation

$$
h_{\Delta}(x)=x+a\left(h_{\Delta}(x)\right) \Delta, \quad x \in \mathbb{R}^{d}
$$

with $\Delta<\Delta_{*}$. Since $h_{\Delta}$ converges to the identity for $\Delta \rightarrow 0$ this perturbed SDE is close to original SDE. To establish Theorem 4.1, it thus remains to show that the split-step backward Euler scheme is close to (15), which can be done along the lines of the proof of Theorem 3.1 . 
If the drift-coefficient is additionally also polynomially Lipschitz, then the standard strong convergence rate $1 / 2$ can even be recovered.

Theorem 4.2. Let the assumptions of Theorem 4.1 hold and assume additionally that there exist $C, q>0$ such that

$$
|a(x)-a(y)| \leq C \cdot\left(1+|x|^{q}+|y|^{q}\right) \cdot|x-y|, \quad x, y \in \mathbb{R}^{d} .
$$

Then, the split-step backward Euler scheme given by (13) and the backward Euler scheme given by (14) are well defined for $\Delta<\Delta^{*}$ and have strong convergence order $1 / 2$, i.e. for both schemes there exists a constant $K>0$ such that

$$
\mathbf{E} \max _{k=0, \ldots, n}\left|X_{t_{k}}-\bar{X}_{t_{k}}^{n}\right|^{2} \leq K \cdot n^{-1}
$$

As pointed out above, in each step of both schemes an implicit equation has to be solved. If the function $h_{\Delta}$ is not known explicitly, this has to be done numerically and may be time-consuming. Solving implicit equations can be avoided by using the so-called tamed Euler method, which has been proposed by Hutzenthaler, Jentzen \& Kloeden in 35]:

$$
\bar{X}_{t_{k+1}}=\bar{X}_{t_{k}}+\frac{1}{1+\left|a\left(\bar{X}_{t_{k}}\right)\right| \Delta} a\left(\bar{X}_{t_{k}}\right) \Delta+\sum_{j=1}^{m} b_{j}\left(\bar{X}_{t_{k}}\right) \Delta_{k} W^{(j)} .
$$

Here the drift-term is "tamed" by the factor $\frac{1}{1+\left|a\left(\bar{X}_{t_{k}}\right)\right| \Delta}$ in the $k$-th step, which prevents a possible explosion of the scheme.

Theorem 4.3. Let the assumptions of Theorem 4.2 hold. Then, there exists a constant $K>0$ such that the tamed Euler scheme given by (16) satisfies

$$
\mathbf{E} \max _{k=0, \ldots, n}\left|X_{t_{k}}-\bar{X}_{t_{k}}^{n}\right|^{2} \leq K \cdot n^{-1}
$$

Here, the difficulty is again to control the moments of the approximation scheme. For this appropriate processes are used that dominate the tamed Euler scheme on subevents whose probabilities converge sufficiently fast to one.

The Theorems given so far in this section require the diffusion coefficient to be globally Lipschitz, which is often not fulfilled in SDEs arising from mathematical finance. However, the backward Euler method can be also successfully applied to the Ait-Sahalia interest rate model

$$
d X_{t}=\left(\alpha_{-1} X_{t}^{-1}-\alpha_{0}+\alpha_{1} X_{t}-\alpha_{2} X_{t}^{r}\right) d t+\sigma X_{t}^{\rho} d W_{t}
$$

where $\alpha_{i}, \sigma>0, i=-1, \ldots, 2$ and $r, \rho>1$. The following result has been obtained by Szpruch et al. in [45]:

Theorem 4.4. Consider the SDE (17) and assume that

$$
r+1>2 \rho .
$$

Then the corresponding backward Euler method (14) with stepsize $\Delta=T / n$ is well defined if $\Delta \leq 1 / \alpha_{1}$, and

$$
\lim _{n \rightarrow \infty} \mathbf{E} \max _{k=0, \ldots, n}\left|X_{t_{k}}-\bar{X}_{t_{k}}^{n}\right|^{2}=0 .
$$


Here the drift coefficient is still one-sided Lipschitz on the domain of the SDE, i.e.

$$
(x-y)(a(x)-a(y)) \leq \alpha_{1}|x-y|^{2}, \quad x, y>0,
$$

and, moreover, $-a$ is coercive on $(0, \infty)$, i.e.

$$
\lim _{x \rightarrow 0} a(x)=\infty \quad \lim _{x \rightarrow \infty} a(x)=-\infty .
$$

These two properties ensure that the drift-implicit Euler scheme for (17) is well-defined and, in particular, takes only strictly positive values.

The drift coefficient in the volatility process

$$
d V_{t}=c_{1} V_{t}\left(c_{2}-V_{t}\right) d t+c_{3} V_{t}^{3 / 2} d W_{t}, \quad V_{0}=v_{0}>0
$$

in the $3 / 2$-model is also one-sided Lipschitz on $(0, \infty)$. It does not, however, satisfy the coercivity assumption. Consequently, the drift-implicit Euler scheme cannot be applied here, since the implicit equation may not be solvable. Note that very recently, Higham et al. introduced in [30] a double-implicit Milstein scheme, which is strongly convergent for the 3/2-model and similar SDEs.

\section{Strong Convergence Rates for the approximation of the CoX-Ingersoll-Ross PROCEss And the Heston MOdel}

Strong convergence rates for the approximation of the CIR process

$$
d X_{t}=\kappa\left(\lambda-X_{t}\right) d t+\theta \sqrt{X_{t}} d W_{t}, \quad t \in[0, T], \quad X_{0}=x_{0}>0
$$

with $\kappa, \lambda, \theta>0$ have been a long standing open problem, even in the regime where the CIR process does not hit zero, i.e. when $2 \kappa \lambda \geq \theta^{2}$.

The first non-logarithmic rates were derived by Berkaoui, Bossy \& Diop for the symmetrized Euler scheme (6)

$$
\bar{X}_{t_{k+1}}=\left|\bar{X}_{t_{k}}+\kappa\left(\lambda-\bar{X}_{t_{k}}\right) \Delta+\theta \sqrt{\bar{X}_{t_{k}}} \Delta_{k} W\right| \text {. }
$$

They showed in [8] that

$$
\mathbf{E} \max _{k=0, \ldots, n}\left|X_{t_{k}}-\bar{X}_{t_{k}}\right|^{2 p} \leq C_{p} \cdot \Delta^{p}
$$

under the assumption

$$
\frac{2 \kappa \lambda}{\theta^{2}}>1+\sqrt{8} \max \left\{\frac{\sqrt{\kappa}}{\theta} \sqrt{16 p-1}, 16 p-2\right\},
$$

where the constant $C_{p}>0$ depends only on $p, \kappa, \lambda, \theta, x_{0}$ and $T$. Strong convergence rates for a drift-implicit Euler-type scheme were recently obtained under mild assumptions by Dereich, Neuenkirch \& Szpruch in [15]. Their key tool is the use of the Lampertitransformation: by the Itô formula, the transformed process $Y_{t}=\sqrt{X}_{t}$ satisfies the SDE

$$
d Y_{t}=\frac{\alpha}{Y_{t}} d t+\beta Y_{t} d t+\gamma d W_{t}, \quad t \geq 0, \quad Y_{0}=\sqrt{x_{0}}
$$

with

$$
\alpha=\frac{4 \kappa \lambda-\theta^{2}}{8}, \quad \beta=-\frac{\kappa}{2}, \quad \gamma=\frac{\theta}{2} .
$$


At first glance this transformation does not help at all, since the drift coefficient of the arising SDE is singular. However,

$$
a(x)=\frac{\alpha}{x}+\beta x, \quad x>0,
$$

satisfies for $\alpha>0, \beta \in \mathbb{R}$ the restricted one-sided Lipschitz condition

$$
(x-y)(a(x)-a(y)) \leq \beta(x-y)^{2}, \quad x, y>0
$$

The drift-implicit Euler method with stepsize $\Delta>0$ in this case is

$$
\bar{Y}_{t_{k+1}}=\bar{Y}_{t_{k}}+\left(\frac{\alpha}{\bar{Y}_{t_{k+1}}}+\beta \bar{Y}_{t_{k+1}}\right) \Delta+\gamma \Delta_{k} W, \quad k=0,1, \ldots
$$

with $\bar{Y}_{0}=\sqrt{x_{0}}$, which has the explicit solution

$$
\bar{Y}_{t_{k+1}}=\frac{\bar{Y}_{t_{k}}+\gamma \Delta_{k} W}{2(1-\beta \Delta)}+\sqrt{\frac{\left(\bar{Y}_{t_{k}}+\gamma \Delta_{k} W\right)^{2}}{4(1-\beta \Delta)^{2}}+\frac{\alpha \Delta}{1-\beta \Delta}} .
$$

Setting

$$
\bar{X}_{t_{k}}=\bar{Y}_{t_{k}}^{2}, \quad k=0,1, \ldots,
$$

gives a positivity preserving approximation of the CIR process, which is called driftimplicit square-root Euler method. This scheme had already been proposed in [4], but without a convergence analysis. Piecewise linear interpolation, i.e.

$$
\bar{X}_{t}=\frac{t_{k+1}-t}{\Delta} \bar{X}_{t_{k}}+\frac{t-t_{k}}{\Delta} \bar{X}_{t_{k+1}}, \quad t \in\left[t_{k}, t_{k+1}\right]
$$

gives a global approximation $\left(\bar{X}_{t}\right)_{t \in[0, T]}$ of the CIR process on $[0, T]$. The main result of [15] is:

Theorem 5.1. Let $2 \kappa \lambda>\theta^{2}, x_{0}>0$ and $T>0$. Then, for all

$$
1 \leq p<\frac{2 \kappa \lambda}{\theta^{2}}
$$

there exists a constant $K_{p}>0$ such that

$$
\left(\mathbf{E} \max _{t \in[0, T]}\left|X_{t}-\bar{X}_{t}\right|^{p}\right)^{1 / p} \leq K_{p} \cdot \sqrt{\log (\Delta) \mid} \cdot \sqrt{\Delta}
$$

for all $\Delta \in(0,1 / 2]$.

The restriction on $p$ arises in the proof of the convergence rate when controlling the inverse $p$-th moments of the CIR process, which are infinite for $p \geq 2 \kappa \lambda / \theta^{2}$. For further details, see [15]. Note that for SDEs with Lipschitz coefficients the convergence rate $\sqrt{|\log (\Delta)|} \cdot \sqrt{\Delta}$ is best possible with respect to the above global error criterion, see [43]. So the convergence rate given in Theorem 5.1 matches the rate that is optimal under standard assumptions.

Other approximation schemes for the strong approximation of the CIR process can be found in [4, 21, 25]. Among them is the drift-implicit Milstein scheme

$$
\bar{Z}_{t_{k+1}}=\bar{Z}_{t_{k}}+\kappa\left(\lambda-\bar{Z}_{t_{k+1}}\right) \Delta+\theta \sqrt{\bar{Z}_{t_{k}}} \Delta_{k} W+\frac{\theta^{2}}{4}\left(\left(\Delta_{k} W\right)^{2}-\Delta\right)
$$


with $Z_{0}=x_{0}$, see [21]. It can be rewritten as

$$
\bar{Z}_{t_{k+1}}=\frac{1}{1+\kappa \Delta}\left(\sqrt{\bar{Z}_{t_{k}}}+\frac{\theta}{2} \Delta_{k} W\right)^{2}+\frac{1}{1+\kappa \Delta}\left(\kappa \lambda-\frac{\theta^{2}}{4}\right) \Delta,
$$

so this scheme preserves the positivity of the CIR process if $4 \kappa \lambda \geq \theta^{2}$. It coincides up to a term of second order with the drift-implicit square-root Euler method, since the latter can be written as

$$
\begin{gathered}
\bar{X}_{t_{k+1}}=\frac{1}{1+\kappa \Delta}\left(\sqrt{\bar{X}_{t_{k}}}+\frac{\theta}{2} \Delta_{k} W\right)^{2}+\frac{1}{1+\kappa \Delta}\left(\kappa \lambda-\frac{\theta^{2}}{4}\right) \Delta \\
-\frac{1}{1+\kappa \Delta}\left(\frac{4 \kappa \lambda-\theta^{2}}{8 \sqrt{\bar{X}_{t_{k+1}}}}-\frac{\kappa}{2} \sqrt{\bar{X}_{t_{k+1}}}\right)^{2} \Delta^{2} .
\end{gathered}
$$

Moreover the drift-implicit Milstein scheme dominates the drift-implicit square-root Euler method:

Lemma 5.2. Let $2 \kappa \lambda>\theta^{2}, x_{0}>0$ and $T>0$. Then

$$
\mathbf{P}\left(\bar{Z}_{t_{k}} \geq \bar{X}_{t_{k}}, k=0,1, \ldots\right)=1 .
$$

Proof. The numerical flow for the drift-implicit Milstein scheme is given by

$$
\varphi_{\bar{Z}}(x ; k, \Delta)=\frac{1}{1+\kappa \Delta}\left(\sqrt{x}+\frac{\theta}{2} \Delta_{k} W\right)^{2}+\frac{1}{1+\kappa \Delta}\left(\kappa \lambda-\frac{\theta^{2}}{4}\right) \Delta
$$

and for the drift-implicit square-root Euler method it satisfies

$$
\begin{aligned}
\varphi_{\bar{X}}(x ; k, \Delta)+\frac{1}{1+\kappa \Delta}\left(\frac{4 \kappa \lambda-\theta^{2}}{8 \sqrt{\varphi_{\bar{X}}(x ; k, \Delta)}}-\frac{\kappa}{2} \sqrt{\varphi_{\bar{X}}(x ; k, \Delta)}\right)^{2} \Delta^{2} \\
=\frac{1}{1+\kappa \Delta}\left(\sqrt{x}+\frac{\theta}{2} \Delta_{k} W\right)^{2}+\frac{1}{1+\kappa \Delta}\left(\kappa \lambda-\frac{\theta^{2}}{4}\right) \Delta .
\end{aligned}
$$

From [4] it is known that $\varphi_{\bar{X}}$ is monotone, i.e.

$$
\varphi_{\bar{X}}\left(x_{1} ; k, \Delta\right) \geq \varphi_{\bar{X}}\left(x_{2} ; k, \Delta\right)
$$

for $x_{1} \geq x_{2}$. Thus it remains to show that

$$
\varphi_{\bar{Z}}(x ; k, \Delta) \geq \varphi_{\bar{X}}(x ; k, \Delta)
$$

for arbitrary $\Delta>0, k=0,1, \ldots, x>0$. However, this follows directly by comparing both flows.

The above property allows one to show the strong convergence of the drift-implicit Milstein scheme, which seems not to have been established yet in the literature.

Proposition 5.3. Let $2 \kappa \lambda>\theta^{2}, x_{0}>0$ and $T>0$. Then

$$
\lim _{n \rightarrow \infty} \mathbf{E} \max _{k=0, \ldots, n}\left|X_{t_{k}}-\bar{Z}_{t_{k}}^{n}\right|^{2}=0 .
$$


Proof. First note that the drift-implicit square-root Euler method can be rearranged as

$$
\begin{aligned}
X_{t_{k+1}}=\varphi_{\bar{Z}} & \left(X_{t_{k}} ; k, \Delta\right)-\kappa \int_{t_{k}}^{t_{k+1}}\left(X_{t}-X_{t_{k+1}}\right) d t \\
& +\theta \int_{t_{k}}^{t_{k+1}}\left(\sqrt{X_{t}}-\sqrt{X_{t_{k}}}\right) d W_{t}-\frac{\theta^{2}}{4}\left(\Delta_{k} W^{2}-\Delta\right)
\end{aligned}
$$

where $\varphi_{\bar{Z}}$ is the numerical flow of the drift-implicit Milstein scheme defined in the proof of the above Lemma. Thus the error $e_{k}=X_{t_{k}}-\bar{Z}_{t_{k}}$ satisfies the recursion

$$
e_{k+1}=e_{k}-\kappa e_{k+1} \Delta+\theta\left(\sqrt{X_{t_{k}}}-\sqrt{\bar{Z}_{t_{k}}}\right) \Delta_{k} W+\rho_{k+1}
$$

with $e_{0}=0$, where

$$
\rho_{k+1}=-\kappa \int_{t_{k}}^{t_{k+1}}\left(X_{s}-X_{t_{k+1}}\right) d s+\theta \int_{t_{k}}^{t_{k+1}}\left(\sqrt{X_{s}}-\sqrt{X_{t_{k}}}\right) d W_{s} .
$$

Now (21) gives

$$
e_{k+1}=\frac{1}{1+\kappa \Delta}\left(e_{k}+\theta\left(\sqrt{X_{t_{k}}}-\sqrt{\bar{Z}_{t_{k}}}\right) \Delta_{k} W+\rho_{k+1}\right)
$$

SO

$$
e_{k}=\sum_{\ell=0}^{k-1} \frac{\theta}{(1+\kappa \Delta)^{k-\ell}}\left(\sqrt{X_{t_{\ell}}}-\sqrt{\bar{Z}_{t_{\ell}}}\right) \Delta_{\ell} W+\sum_{\ell=0}^{k-1} \frac{1}{(1+\kappa \Delta)^{k-\ell}} \rho_{\ell+1} .
$$

Straightforward calculations using (20) yield

$$
\sup _{n \in \mathbb{N}} \sup _{k=0, \ldots, n} \mathbf{E} \bar{Z}_{t_{k}}<\infty
$$

Then applying the Burkholder-Davis-Gundy inequality to the martingale

$$
M_{k}=\sum_{\ell=0}^{k-1}(1+\kappa \Delta)^{\ell}\left(\sqrt{X_{t_{\ell}}}-\sqrt{\bar{Z}_{t_{\ell}}}\right) \Delta_{\ell} W, \quad k=0,1, \ldots
$$

gives

$$
\begin{aligned}
\mathbf{E} \sup _{k=0, \ldots, n} e_{k}^{2} \leq c \sum_{\ell=0}^{n-1}(1+\kappa \Delta)^{2 \ell} \mathbf{E}\left|\sqrt{X_{t_{\ell}}}-\sqrt{\bar{Z}_{t_{\ell}}}\right|^{2} \Delta \\
\quad+c \mathbf{E} \sup _{k=1, \ldots, n}\left|\sum_{\ell=0}^{k-1} \frac{1}{(1+\kappa \Delta)^{k-\ell}} \rho_{\ell+1}\right|^{2} .
\end{aligned}
$$

Here and below constants whose particular value is not important will be denoted by $c$ regardless of their value.

It remains to estimate the terms on the right side of the equation (22). The previous Lemma implies that

$$
\mathbf{E}\left|\bar{Z}_{t_{k}}-\bar{X}_{t_{k}}\right|=\mathbf{E}\left(\bar{Z}_{t_{k}}-\bar{X}_{t_{k}}\right)
$$

SO

$$
\mathbf{E}\left|\bar{Z}_{t_{k}}-X_{t_{k}}\right| \leq 2 \mathbf{E}\left|\bar{X}_{t_{k}}-X_{t_{k}}\right|+\left|\mathbf{E}\left(\bar{Z}_{t_{k}}-X_{t_{k}}\right)\right| .
$$

Clearly, Theorem 5.1 yields

$$
\max _{k=0, \ldots, n} \mathbf{E}\left|\bar{X}_{t_{k}}-X_{t_{k}}\right| \leq c \cdot \sqrt{|\log (\Delta)|} \cdot \sqrt{\Delta}
$$


Moreover,

$$
\mathbf{E} \bar{Z}_{t_{k+1}}=\mathbf{E} \bar{Z}_{t_{k}}+\kappa\left(\lambda-\mathbf{E} \bar{Z}_{t_{k+1}}\right) \Delta
$$

which is the drift-implicit Euler approximation of

$$
\mathbf{E} X_{t}=x_{0}+\int_{0}^{t} \kappa\left(\lambda-\mathbf{E} X_{s}\right) d s, \quad t \in[0, T],
$$

at the discretization points $t_{k}=k \Delta$, so

$$
\max _{k=0, \ldots, n}\left|\mathbf{E}\left(X_{t_{k}}-\bar{Z}_{t_{k}}\right)\right| \leq c \cdot \Delta \text {. }
$$

Hence

$$
\max _{k=0, \ldots, n} \mathbf{E}\left|\bar{X}_{t_{k}}-X_{t_{k}}\right| \leq c \cdot \sqrt{|\log (\Delta)|} \cdot \sqrt{\Delta}
$$

which gives

$$
\sum_{k=0}^{n}(1+\kappa \Delta)^{2 k} \mathbf{E}\left|\sqrt{X_{t_{k}}}-\sqrt{\bar{Z}_{t_{k}}}\right|^{2} \Delta \leq c \cdot \sqrt{|\log (\Delta)|} \cdot \sqrt{\Delta}
$$

since $|\sqrt{x}-\sqrt{y}| \leq \sqrt{|x-y|}$ for $x, y>0$ and $\sup _{n \in \mathbb{N}} \sup _{k=0, \ldots, n}(1+\kappa \Delta)^{2 k}<\infty$.

For the second term, applying the Burkholder-Davies-Gundy inequality and Jensen's inequality yield

$$
\begin{aligned}
& \mathbf{E} \sup _{k=1, \ldots, n}\left|\sum_{\ell=0}^{k-1} \frac{1}{(1+\kappa \Delta)^{k-\ell}} \rho_{\ell+1}\right|^{2} \\
& \quad \leq c \cdot \frac{1}{\Delta} \sum_{k=0}^{n-1} \mathbf{E}\left|\int_{t_{k}}^{t_{k+1}}\left(X_{t}-X_{t_{k+1}}\right) d t\right|^{2}+c \sum_{k=0}^{n-1} \mathbf{E} \int_{t_{k}}^{t_{k+1}}\left|\sqrt{X_{t}}-\sqrt{X_{t_{k}}}\right|^{2} d t .
\end{aligned}
$$

Now

so it follows that

$$
\mathbf{E}\left|X_{t}-X_{s}\right|^{2} \leq c \cdot|t-s|, \quad s, t \in[0, T]
$$

$$
\mathbf{E} \sup _{k=1, \ldots, n}\left|\sum_{\ell=0}^{k-1} \frac{1}{(1+\kappa \Delta)^{k-\ell}} \rho_{\ell+1}\right|^{2} \leq c \cdot \sqrt{\Delta},
$$

which completes the proof of the proposition.

Alternatively, Proposition 5.3 could have been obtained by deriving the pathwise convergence of the drift-implicit Milstein scheme and establishing the uniform integrability of the squared maximum error. Note that the above proof gives also the convergence order $1 / 4$ up to a logarithmic term. However this rate seems to be suboptimal, see the following numerical example.

Example 5.4. The Figures 4 and 5 show the empirical root mean square maximum error in the discretization points, i.e.

$$
\left(\frac{1}{N} \sum_{i=1}^{N} \max _{k=0, \ldots, n}\left|X_{t_{k}}^{*,(i)}-\bar{X}_{t_{k}}^{n,(i)}\right|^{2}\right)^{1 / 2}
$$

versus the step size for the approximation of the CIR process. Consider the 
(i) truncated Euler scheme (2)

(ii) drift-implicit square-root Euler (19)

(iii) drift-implicit Milstein scheme (20)

for the Scenarios I and II (see Example 2.3). Scenario I satisfies the condition of Theorem 5.1 and Proposition 5.3 since $2 \kappa \lambda / \theta^{2}=2.011276 \ldots$. This condition is violated in Scenario II where $2 \kappa \lambda / \theta^{2}=0.36$. In the latter scenario, the truncation $\sqrt{x^{+}}$in the definition of the schemes (ii) and (iii) is used, since both discretization schemes may take negative values here. The numerical reference solution $X^{*}$ is computed in Scenario I using scheme (ii) with very small stepsize and in Scenario II with scheme (i) with a very small stepsize. The number of repetitions of the Monte Carlo simulation is $N=5 \cdot 10^{4}$. In the $\log$ -

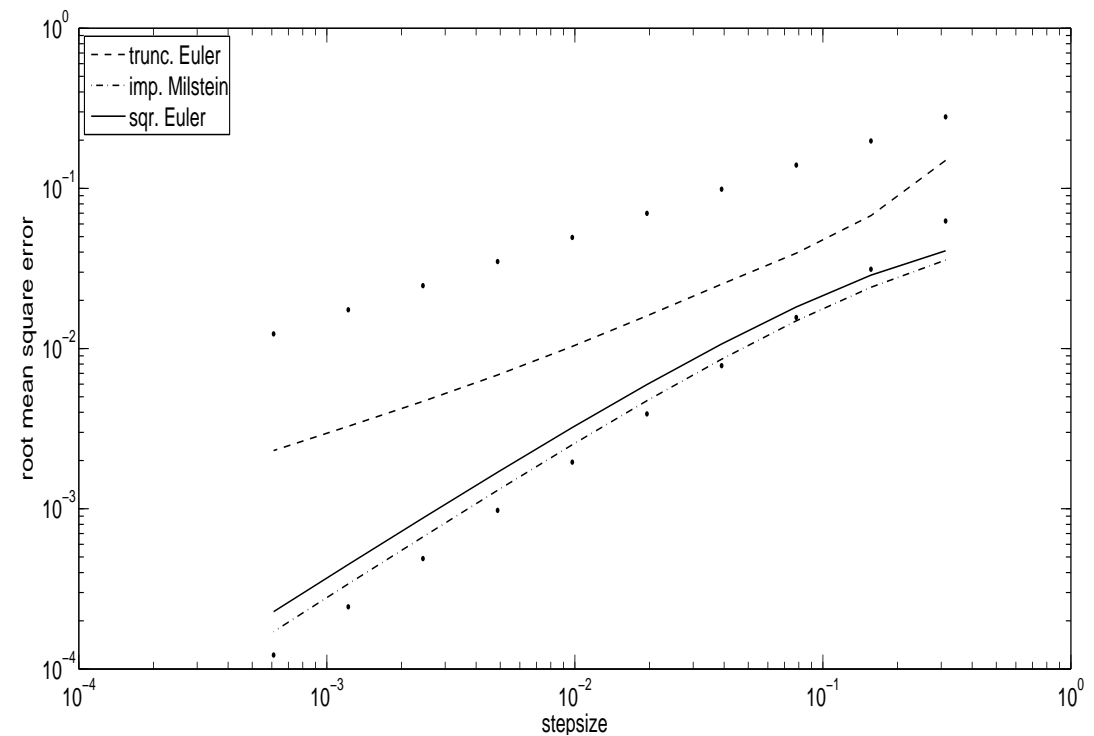

FiguRE 4. Root mean square errors vs. step size for Scenario I of the CIR process

log coordinates here, the dots indicate the convergence orders 0.5 and 1.0 in Figure 4 and 0.25 and 0.5 in Figure 5, respectively. For Scenario I the empirical mean square error for the truncated Euler scheme seems to decay with the order 0.5, while the other schemes seem to have an empirical convergence order close to 1.0. (For smooth and Lipschitz coefficients the Milstein scheme is of order one for the maximum error in the discretization points.)

For Scenario II, these convergence orders deteriorate and for all schemes are significantly lower than one half, see also the following table, where the convergence orders have been estimated by a linear regression.

\begin{tabular}{c||c|c|c|c|} 
empirical conv. order / for & Sc. I 'part' & Sc. II 'part' & Sc. I 'full' & Sc. II 'full' \\
\hline \hline truncated Euler & 0.5739 & 0.3193 & 0.6446 & 0.2960 \\
\hline drift-imp. square-root Euler & 0.9281 & 0.2734 & 0.8491 & 0.2837 \\
\hline drift-imp. Milstein & 0.9447 & 0.3096 & 0.8719 & 0.2871
\end{tabular}

Here 'part' denotes the results for the linear regression using only the step sizes $\Delta=$ $5 \cdot 2^{-j}, j=7, \ldots, 13$, while 'full' uses the full data set, i.e. the step sizes $\Delta=5 \cdot 2^{-j}$, $j=4, \ldots, 13$. 


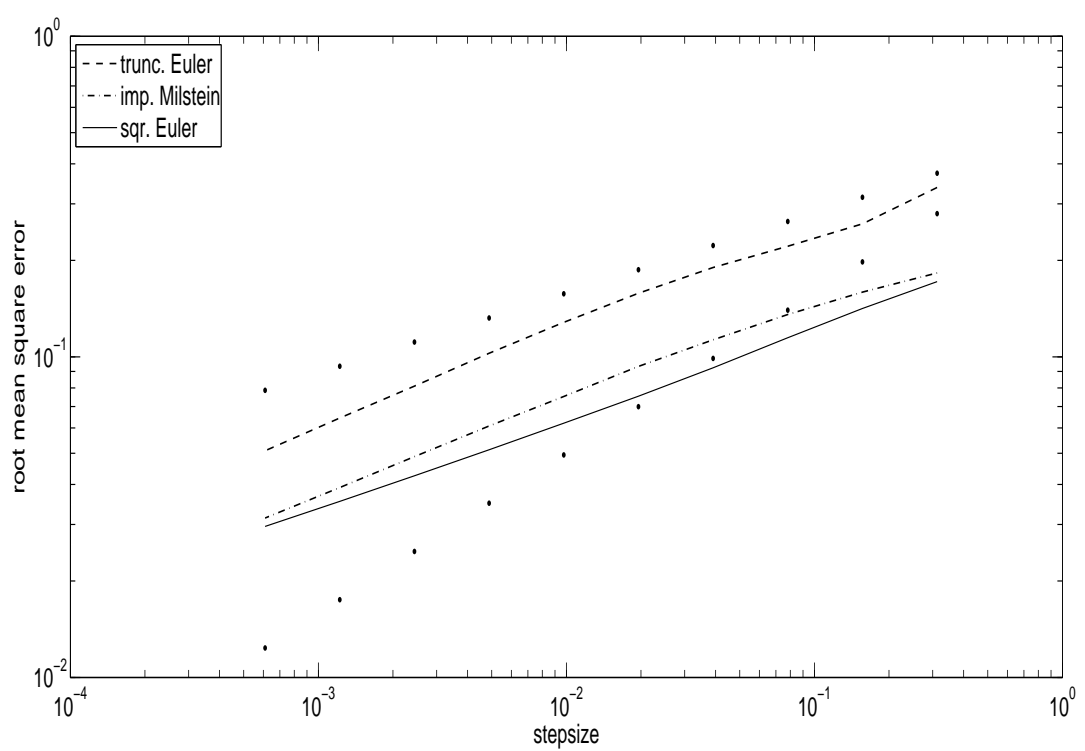

Figure 5. Root mean square errors vs. step size for Scenario II of the CIR process

Applying the Lamperti-transformation also to the asset price in the Heston model gives the log-Heston model

$$
\begin{array}{rlrl}
d \log \left(S_{t}\right) & =\left(\mu-\frac{1}{2} Y_{t}^{2}\right) d t+Y_{t}\left(\sqrt{1-\rho^{2}} d W_{t}^{(1)}+\rho d W_{t}^{(2)}\right), & S_{0} & =s_{0}>0 \\
d Y_{t} & =\left(\frac{4 \kappa \lambda-\theta^{2}}{8} \frac{1}{Y_{t}}-\frac{\kappa}{2} Y_{t}\right) d t+\frac{\theta}{2} d W_{t}^{(2)}, & Y_{0}=y_{0}>0 .
\end{array}
$$

The approximation of the log-Heston price is then a simple integration problem. Using the Euler scheme for the log-price equation and the drift-implicit square-root Euler scheme for the volatility process yields an approximation $\bar{H}_{t_{k}}$ of $\log \left(S_{t_{k}}\right)$ given by

$$
\bar{H}_{t_{k}}=\log \left(s_{0}\right)+\sum_{\ell=0}^{k-1}\left(\mu-\frac{1}{2} \bar{Y}_{t_{\ell}}^{2}\right) \Delta+\sum_{\ell=0}^{k-1} \bar{Y}_{t_{\ell}}\left(\sqrt{1-\rho^{2}} \Delta_{\ell} W^{(1)}+\rho \Delta_{\ell} W^{(2)}\right) .
$$

This is extended by piecewise linear interpolation to $[0, T]$.

Corollary 5.5. Let $2 \kappa \lambda>\theta^{2}, x_{0}>0$ and $T>0$. Then, for all

$$
1 \leq p<\frac{2 \kappa \lambda}{\theta^{2}}
$$

there exists a constant $K_{p}>0$ such that

$$
\left(\mathbf{E} \max _{t \in[0, T]}\left|\log \left(S_{t}\right)-\bar{H}_{t}\right|^{p}\right)^{1 / p} \leq K_{p} \cdot \sqrt{|\log (\Delta)|} \cdot \sqrt{\Delta}
$$

for all $\Delta \in(0,1 / 2]$. 
Note that in the Heston model moment explosions may appear according to the parameters of the SDE. In particular, for $p>1$ one has $\mathbf{E} S_{t}^{p}<\infty$ for all $t>0$ if and only if

$$
\rho \leq-\frac{\sqrt{p-1}}{\sqrt{p}}+\frac{\kappa}{\theta p}
$$

For more details see e.g. [6]. Whether this phenomenon also arises for discretization schemes for the Heston model is unknown at the time of writing.

Example 5.6. In this example we test the efficiency of the Multi-level Monte Carlo estimator $\widehat{P}_{m l}$ see [19, 20], based on the above approximation scheme for the valuation of a European Call option, i.e. for

$$
p=e^{-r T} \mathbf{E}\left(S_{T}-K\right)^{+} .
$$

The parameters for the Heston model are

$$
\begin{aligned}
& v_{0}=0.05, \quad \kappa=5.07, \quad \lambda=0.0457, \quad \theta=0.48, \quad T=1 \\
& s_{0}=100, \quad \mu=r=0.0319, \quad \rho=-0.7, \quad K=105 .
\end{aligned}
$$

(Since the riskfree measure is used for the valuation we have $\mu=r$.) In view of the above convergence result for the log-Heston model, we use the number of levels $L=$ $\left\lceil\log _{2}\left(T \varepsilon^{-1}\right)\right\rceil$ and the number of repetitions $N_{l}=\left\lceil L \varepsilon^{-2} T 2^{-\ell}\right\rceil, \ell=0, \ldots, L$, for a given input accuracy $\varepsilon>0$, see [19].

The table below shows the empirical root mean square error

$$
\operatorname{rmsq}=\sqrt{\frac{1}{M} \sum_{i=1}^{M}\left|p-\widehat{P}_{m l}^{(i)}\right|^{2}}
$$

for the Multi-level estimator versus the required number of total Euler steps. The latter is proportional to the overall computational cost of the estimator, i.e. the number of used random numbers, number of function evaluations and number of arithmetic operations. The $\widehat{P}_{m l}^{(i)}$ are iid copies of the Multi-level estimator $\widehat{P}_{m l}$ and we use $M=5 \cdot 10^{4}$. The reference value $p=7.46253$ was obtained by a numerical evaluation of its Fourier transform representation, see e.g. [3].

For comparison, we also provide the corresponding numerical data for the standard Monte Carlo estimator $\widehat{P}_{s t}$, see (11), for which we use the relation $\Delta^{2}=T / N$ to match stepsize $\Delta$ and numbers of repetitions $N$. For the same parameters as above the empirical root mean square error of $\widehat{P}_{s t}$ is again estimated using $M=5 \cdot 10^{4}$ repetitions.

\begin{tabular}{c||r|r||r|r|}
$\varepsilon$ & Euler steps of $\widehat{P}_{m l}$ & $\mathrm{rmsq}_{\mathrm{emp}}$ of $\widehat{P}_{m l}$ & Euler steps of $\widehat{P}_{s t}$ & $\mathrm{rmsq}_{\mathrm{emp}}$ of $\widehat{P}_{s t}$ \\
\hline \hline $2^{-3}$ & 1056 & 1.369616 & 512 & 1.444497 \\
\hline $2^{-4}$ & 7168 & 0.685299 & 4096 & 0.714207 \\
\hline $2^{-5}$ & 43520 & 0.352762 & 32768 & 0.357962 \\
\hline $2^{-6}$ & 245760 & 0.181384 & 262144 & 0.179231 \\
\hline $2^{-7}$ & 1318912 & 0.093485 & 2097152 & 0.089618 \\
\hline $2^{-8}$ & 6815744 & 0.047139 & 16777216 & 0.044821 \\
\hline
\end{tabular}

The numerical data are in good accordance with the predicted convergence behavior, that is 
- for the Multi-level estimator a root mean square error of order $\varepsilon$ for a computational cost of order $\varepsilon^{-2}|\log (\varepsilon)|^{2}$

- and for the standard estimator a root mean square error of order $\varepsilon$ for a computational cost of order $\varepsilon^{-3}$.

In particular halving the input accuracy leads for both estimators (approximately) to a halving of the empirical root mean square error. Moreover, these results illustrate nicely the superiority of the Multi-level estimator for small input accuracies.

\section{Summary and Outlook}

In this article we gave a survey on recent results on the convergence of numerical methods for stochastic differential equations in mathematical finance. The presented results include:

- the pathwise convergence of general Itô-Taylor schemes for strictly positive SDEs with smooth but not globally Lipschitz coefficients (Section 2);

- the construction of structure, i.e. positivity, preserving approximation schemes (Sections 2 and 5);

- the strong convergence of Euler-type methods for the CEV model and the CIR process (Section 3);

- the explosion of the moments of the Euler scheme for SDEs for the 3/2-model (Section 3);

- the strong convergence of the drift-implicit Euler scheme for the Ait-Sahalia model (Section 4);

- strong convergence rates for the approximation of the CIR and the log-Heston model using a drift-implicit Euler-type method (Section 5).

However many unsettled questions are remaining: the exact strong convergence rate of the Euler scheme for the CEV and CIR processes, the existence or non-existence of moment explosions for approximation schemes of the Heston model, how to prevent moment explosions (if they happen) by simple modifications of the scheme etc. And even if these questions are answered, the question remains whether there is a 'general theory' for numerical methods for SDEs from mathematical finance or do these SDEs have to analysed one by one. So, the numerical analysis of SDEs arising in finance will be still an active and challenging field of research in the future.

Acknowledgements. The authors would like to thank Martin Altmayer, Martin Hutzenthaler and Arnulf Jentzen for valuable comments and remarks on an earlier version of the manuscript. Moreover, the authors would like to thank Mike Giles for a helpful discussion concerning the numerical evaluation of Fourier transforms. 


\section{REFERENCES}

[1] Ait-Sahalia, A.: Testing continuous-time models of the spot interest rate. Rev. Financ. Stud. 9, no. 2, 385-426 (1996)

[2] Ait-Sahalia, A., Kimmel, R.: Maximum Likelihood Estimation of Stochastic Volatility Models. J. Financ. Econ. 83, 413-452 (2007)

[3] Albrecher, H., Mayer, Ph., Schoutens, W., Tistaert, J.: The Little Heston Trap. Wilmott Magazine, January Issue, 83-92. (2007)

[4] Alfonsi, A.: On the discretization schemes for the CIR (and Bessel squared) processes. Monte Carlo Methods Appl. 11, 355-384 (2005)

[5] Alfonsi, A.: High order discretization schemes for the CIR process: Application to affine term structure and Heston models. Math. Comput. 79, no. 269, 209-237 (2010)

[6] Andersen, L., Piterbarg, V.: Moment explosions in stochastic volatility models. Finance Stoch. 11, no. 1, 29-50 (2007)

[7] Arnold, L.: Random Dynamical Systems, Springer, Berlin (1998)

[8] Berkaoui, A., Bossy, M., Diop, A.: Euler scheme for SDEs with non-Lipschitz diffusion coefficient: strong convergence. ESAIM, Probab. Stat. 12, 1-11 (2008)

[9] Beskos, A., Papaspiliopoulos, O., Roberts G.: Retrospective exact simulation of diffusion sample paths with applications. Bernoulli 12, no. 6, 1077-1098 (2006)

[10] Bossy, M., Diop, A.: An efficient discretization scheme for one dimensional SDEs with a diffusion coefficient function of the form $|x|^{a}, a \in[1 / 2,1)$. Working paper, INRIA (2007)

[11] Broadie, M., Kaya, Ö.: Exact simulation of stochastic volatility and other affine jump diffusion processes. Oper. Res. 54, 217-231 (2006)

[12] Cox, J.: Notes on option pricing I: Constant elasticity of variance diffusions. Working paper, Stanford University (1975)

[13] Cox, J., Ingersoll, J., Ross, S.: A theory of the term structure of interest rates. Econometrica 53 , 385-408 (1985)

[14] Deelstra, G., Delbaen, F.: Convergence of discretized stochastic (interest rate) processes with stochastic drift term. Appl. Stochastic Models Data Anal. 14, 77-84 (1998)

[15] Dereich, S., Neuenkirch, A., Szpruch, L.: An Euler-type method for the strong approximation of the Cox-Ingersoll-Ross process. Proc. Roy. Soc. A, to appear

[16] Deuflhard, P., Bornemann, F.: Scientific computing with ordinary differential equations. Springer, New York (2002)

[17] Garrido-Atienza, M.J., Kloeden, P.E., Neuenkirch, A.: Discretization of stationary solutions of stochastic systems driven by fractional Brownian motion, J. Appl Math. Optim. 60, 151-172 (2009)

[18] Duffie, D., Glynn, P.: Efficient Monte Carlo simulation of security prices. Ann. Appl. Probab. 5, no. 4, 897-905 (1995)

[19] Giles, M.: Multi-level Monte Carlo path simulation. Oper. Res. 56, no. 3, 607-617 (2008)

[20] Giles, M.: Improved multilevel Monte Carlo convergence using the Milstein scheme. In: Keller, A. (ed.) et al., Monte Carlo and Quasi-Monte Carlo Methods 2006, Proceedings. Berlin, Springer, $343-354$ (2007)

[21] Günther, M., Kahl, C., Roßberg, T.: Structure preserving stochastic integration schemes in interest rate derivative modeling. Appl. Numer. Math. 58, no. 3, 284-295 (2008)

[22] Makarov, R., Glew, D.: Exact simulation of Bessel diffusions. Monte Carlo Methods Appl. 16, no. 3-4, 283-306 (2010)

[23] Gyöngy, I.: A note on Euler's approximations, Potential Anal. 8, 205-216 (1998)

[24] Gyöngy, I., Rásonyi, M.: A note on Euler approximations for SDEs with Hölder continuous diffusion coefficients, Stochastic Processes Appl. 121, no. 10, 2189-2200 (2011)

[25] Halidas, H.: Semi discrete approximations for stochastic differential equations and applications. Int. J. Comput. Math. 89, no. 6, 780-794 (2012)

[26] Heston, S.: A closed form solution for options with stochastic volatility, with applications to bonds and currency options. Rev. Financial Stud. 6, 327-343 (1993)

[27] Heston, S.: A simple new formula for options with stochastic volatility. Working paper, Washington University of St. Louis (1997) 
[28] Higham, D., Mao, X.: Convergence of Monte Carlo simulations involving the mean-reverting square root process. J. Comp. Fin. 8, 35-62 (2005)

[29] Higham, D., Mao, X., Stuart, A.: Strong convergence of Euler-type methods for nonlinear stochastic differential equations. SIAM J. Numer. Anal. 40, 1041-1063 (2002)

[30] Higham, D., Mao, X., Szpruch, L.: Convergence, Non-negativity and Stability of a New Milstein Scheme with Applications to Finance. Working paper (2012) arXiv:1204.1647

[31] Hurd, T.R., Kuznetsov, A.: Explicit formulas for Laplace transforms of stochastic integrals. Markov Process. Relat. Fields 14, no. 2, 277-290 (2008)

[32] Hutzenthaler, M., Jentzen, A.: Convergence of the stochastic Euler scheme for locally Lipschitz coefficients. Found. Comput. Math. 11, no. 6, 657-706 (2011)

[33] Hutzenthaler, M., Jentzen, A.: Numerical approximations of stochastic differential equations with non-globally Lipschitz continuous coefficients. Working paper (2012) arXiv:1203.5809

[34] Hutzenthaler, M., Jentzen A., Kloeden, P.: Strong and weak divergence in finite time of Euler's method for stochastic differential equations with non-globally Lipschitz coefficients. Proc. Roy. Soc. London A 467, no. 2130, 1563-1576 (2011)

[35] Hutzenthaler, M., Jentzen A., Kloeden, P.: Strong convergence of an explicit numerical method for SDEs with non-globally Lipschitz continuous coefficients. Ann. Appl. Probab, to appear

[36] Hutzenthaler, M., Jentzen A., Kloeden, P.: Divergence of the multilevel Monte Carlo Euler method for nonlinear stochastic differential equations. Working paper (2011) arXiv:1105.0226

[37] Jentzen, A., Kloeden, P., Neuenkirch, A.: Convergence of numerical approximations of stochastic differential equations on domains: higher order convergence rates without global Lipschitz coefficients. Numer. Math. 112, no. 1, 41-64 (2009)

[38] Kloeden, P., Neuenkirch, A.: The pathwise convergence of approximation schemes for stochastic differential equations. LMS J. Comput. Math. 10, 235-253 (2007)

[39] Kloeden, P., Platen, E.: Numerical Solution of Stochastic Differential Equations. 3rd edn, Springer, Berlin (1999)

[40] Lord, R., Koekkoek, R., van Dijk, D.: A comparison of biased simulation schemes for stochastic volatility models. Quant. Finan. 10, no. 2, 177-194 (2010)

[41] Milstein, G.: Numerical Integration of Stochastic Differential Equations. Kluwer, Doordrecht (1995)

[42] Milstein, G., Tretyakov, M.: Numerical integration of stochastic differential equations with nonglobally Lipschitz coefficients. SIAM J. Numer. Anal. 43, 1139-1154 (2005)

[43] Müller-Gronbach, T.: The optimal uniform approximation of systems of stochastic differential equations. Ann. Appl. Probab. 12, no. 2, 664-690 (2002)

[44] Neuenkirch, A., Zähle, H.: Asymptotic error distribution of the Euler method for SDEs with non-Lipschitz coefficients. Monte Carlo Methods Appl. 15, no.4, 335-353 (2009)

[45] Szpruch, L., Mao, X., Higham, D., Pan, J.: Numerical simulation of a strongly nonlinear AitSahalia-type interest rate model. BIT 51, 405-425 (2011)

Peter Kloeden, Institut für Mathematik, Johann Wolfgang Goethe-Universität, Robert-Mayer-Strasse 10, D-60325 Frankfurt am Main, GERmany, kloeden@math.uni-frankfurt.de

Andreas Neuenkirch, Institut für Mathematik, Universität Mannheim, A5,6, D-68131

MANnheIM, GERMAnY, neuenkirch@kiwi.math.uni-mannheim.de 